\title{
Antibacterial $p$-Terphenyl with a Rare 2,2'-Bithiazole Substructure and Related Compounds Isolated from the Marine-Derived Actinomycete Nocardiopsis sp. HDN154086
}

Yimin Chang, ${ }^{{ }^{\dagger}}{ }^{\dagger}$ Qian Che,${ }^{\perp \dagger}$ Li Xing, ${ }^{\dagger}$ Chuanteng Ma, ${ }^{\dagger}$ Yaxin Han, ${ }^{\dagger}$ Tianjiao Zhu, ${ }^{\dagger}$

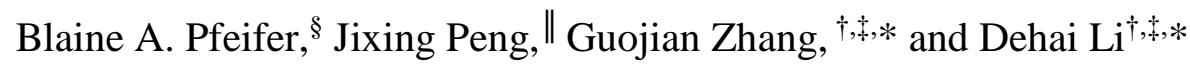

${ }^{\dagger}$ School of Medicine and Pharmacy, Ocean University of China, Qingdao 266003, People's Republic of China

Laboratory for Marine Drugs and Bioproducts of Qingdao National Laboratory for Marine Science and Technology, Qingdao, 266237, People's Republic of China

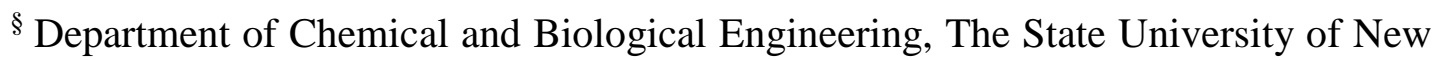
York at Buffalo, Buffalo, NY 14260, United States

$\|$ Key Laboratory of Testing and Evaluation for Aquatic Product Safety and Quality, Ministry of Agriculture and Rural Affairs, P. R. China; Yellow Sea Fisheries Research Institute, Chinese Academy of Fishery Sciences, Qingdao 266071, China

* Corresponding authors. Tel: 0086-532-82031619. Fax: 0086-532-82033054.

E-mail: dehaili@ouc.edu.cn (D. Li); zhangguojian@ouc.edu.cn (G. Zhang). 


\section{List of Supporting Information}

Figure S1. HPLC analysis of the crude extract of Nocardiopsis sp. HDN154086............. 4

Figure S2. The 16S rRNA sequences data of Nocardiopsis sp. HDN154086................... 4

Figure S3. Detailed molecular network of Nocardiopsis sp. HDN154086...................... 5

Figure S4. The MS/MS spectra of the compounds......................................................... 5

Figure S5. ${ }^{1} \mathrm{H}$ NMR $\left(500 \mathrm{MHz}, \mathrm{CDCl}_{3}\right)$ spectrum of nocarterphenyl $\mathrm{D}(\mathbf{1})$................... 7

Figure S6. ${ }^{13} \mathrm{C}$ NMR $\left(125 \mathrm{MHz}, \mathrm{CDCl}_{3}\right)$ spectrum of nocarterphenyl $\mathrm{D}(\mathbf{1})$................. 7

Figure S7. DEPT (125 MHz, $\mathrm{CDCl}_{3}$ ) spectrum of nocarterphenyl D (1) ........................ 8

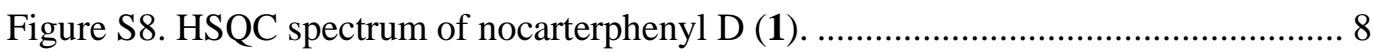

Figure S9. ${ }^{1} \mathrm{H}-{ }^{1} \mathrm{H}$ COSY spectrum of nocarterphenyl D (1) ........................................ 9

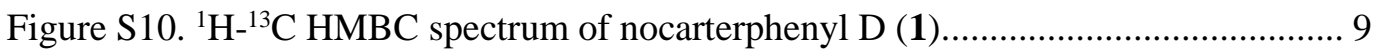

Figure S11. HRESIMS spectrum of nocarterphenyl D (1) ............................................ 10

Figure S12. IR spectrum of nocarterphenyl D (1) ................................................... 10

Figure S13. ${ }^{1} \mathrm{H}$ NMR $\left(500 \mathrm{MHz}, \mathrm{CDCl}_{3}\right)$ spectrum of nocarterphenyl E (2)................. 11

Figure S14. ${ }^{13} \mathrm{C}$ NMR $\left(150 \mathrm{MHz}, \mathrm{CDCl}_{3}\right)$ spectrum of nocarterphenyl E (2)................ 11

Figure S15. DEPT (150 MHz, $\left.\mathrm{CDCl}_{3}\right)$ spectrum of nocarterphenyl E (2) .................... 12

Figure S16. HSQC spectrum of nocarterphenyl E (2) ................................................. 12

Figure S17. ${ }^{1} \mathrm{H}-{ }^{1} \mathrm{H}$ COSY spectrum of nocarterphenyl E (2) ....................................... 13

Figure S18. ${ }^{1} \mathrm{H}-{ }^{13} \mathrm{C}$ HMBC spectrum of nocarterphenyl E (2).................................... 13

Figure S19. HRESIMS spectrum of nocarterphenyl E (2)............................................. 14

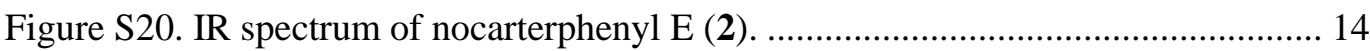

Figure S21. ${ }^{1} \mathrm{H}$ NMR (600 MHz, DMSO) spectrum of nocarterphenyl F (3)................ 15

Figure S22. ${ }^{13} \mathrm{C}$ NMR (150 MHz, DMSO) spectrum of nocarterphenyl F (3)............... 15

Figure S23. DEPTQ $\left(150 \mathrm{MHz}, \mathrm{CDCl}_{3}\right)$ spectrum of nocarterphenyl $\mathrm{F}(\mathbf{3}) \ldots \ldots \ldots \ldots \ldots \ldots . . . . . . . .16$

Figure S24. HSQC spectrum of nocarterphenyl F (3) ................................................. 16

Figure S25. ${ }^{1} \mathrm{H}-{ }^{1} \mathrm{H}$ COSY spectrum of nocarterphenyl F (3) ...................................... 17

Figure S26. ${ }^{1} \mathrm{H}^{-13} \mathrm{C}$ HMBC spectrum of nocarterphenyl $\mathrm{F}(\mathbf{3})$....................................... 17

Figure S27. HRESIMS spectrum of nocarterphenyl F (3)......................................... 18

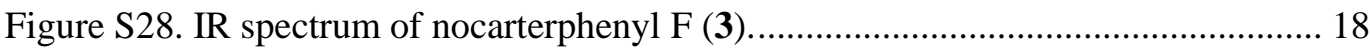

Figure S29. ${ }^{1} \mathrm{H}$ NMR $\left(600 \mathrm{MHz}, \mathrm{CDCl}_{3}\right)$ spectrum of nocarterphenyl G (4) ............... 19

Figure S30. ${ }^{13} \mathrm{C}$ NMR $\left(150 \mathrm{MHz}, \mathrm{CDCl}_{3}\right)$ spectrum of nocarterphenyl $\mathrm{G}(\mathbf{4})$............... 19 
Figure S31. DEPT (150 MHz, $\left.\mathrm{CDCl}_{3}\right)$ spectrum of nocarterphenyl $\mathrm{G}(\mathbf{4})$.

Figure S32. HSQC spectrum of nocarterphenyl G (4) .............................................. 20

Figure S33. ${ }^{1} \mathrm{H}-{ }^{1} \mathrm{H}$ COSY spectrum of nocarterphenyl G (4) .................................... 21

Figure S34. ${ }^{1} \mathrm{H}^{-13} \mathrm{C}$ HMBC spectrum of nocarterphenyl G (4)................................... 21

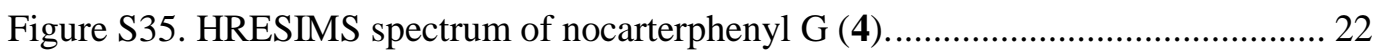

Figure S36. IR spectrum of nocarterphenyl G (4) ……............................................. 22

Figure S37. ${ }^{1} \mathrm{H}$ NMR (400 MHz, $\mathrm{CDCl}_{3}$ ) spectrum of nocarterphenyl H (5), ............... 23

Figure S38. ${ }^{13} \mathrm{C}$ NMR $\left(125 \mathrm{MHz}, \mathrm{CDCl}_{3}\right)$ spectrum of nocarterphenyl $\mathrm{H}(\mathbf{5})$............... 23

Figure S39. HSQC spectrum of nocarterphenyl H (5).............................................. 24

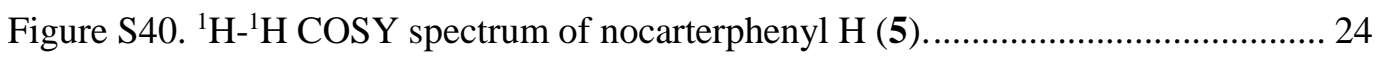

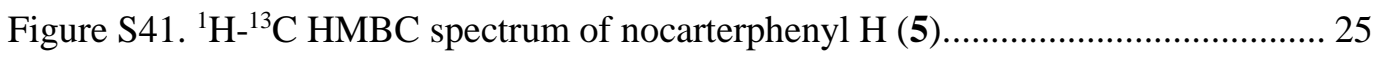

Figure S42. HRESIMS spectrum of nocarterphenyl H (5).......................................... 25

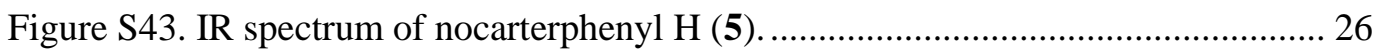

Figure S44. Key HMBC and COSY correlations of nocarterphenyl H (5).................... 26

Figure S45. Plausible biogenetic pathway of nocarterphenyls D-H (1-5)................... 26

Table S1. ${ }^{13} \mathrm{C}$ NMR of 5-methoxy-4,7-bis(4-methoxyphenyl)benzo[ $\left.d\right]$ thiazol-6-ol and nocarterphenyl D (1) in $\mathrm{CDCl}_{3}(\delta$ in ppm) 
Figure S1. HPLC analysis of the crude extract of Nocardiopsis sp. HDN154086.

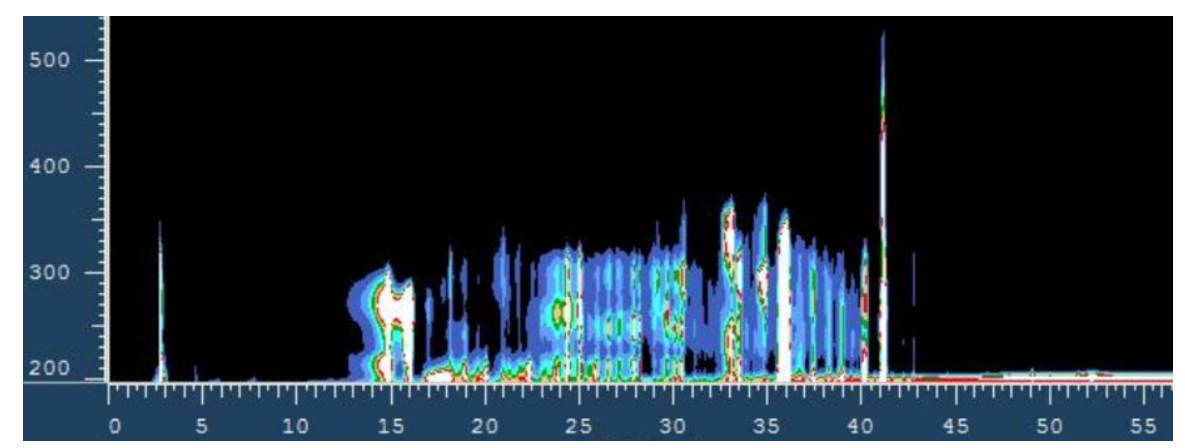

Figure S2. The 16S rRNA sequences data of Nocardiopsis sp. HDN154086.

ACGTCACCTTCCCCACTCCCCCCAGCAAGCTGGTTAGGCCGCAGGTTTCGGGTGTTGC CGACTTTCATGACGTGACGGGCGGTGTGTACAAGGCCCGGGAACGTATTCACCGCGGC ACTGCTGATCCGCGATTACTAGCGACTCCACCTTCATGGGGTCGAGTTGCAGACCCCAA TCCGAACTGAGACCGGCTTTTAGGGATTCGCTCCACCTCACGGCATCGCACGCCCACT GTACCGGCCATTGTAGCATGTTTGCAGCCCAAGACATAAGGGGCATGATGACTTGACGT CATCCCCACCTTCCTCCGAGTTGACCCCGGCAGTCTCCCATGAGTCCCCACCATCACGT GCTGGCAACATGGAATAAGGGTTGCGCTCGTTGCGGGACTTAACCCAACATCTCACGA CACGAGCTGACGACAGCCATGCACCACCTGTCACCGATCCCAAAAGGACCCGCTATCT CTAACGGATTACCGGTGATGTCAAACCTTGGTAAGGTTCTTCGCGTTGCGTCGAATTAA GCAACATGCTCCGCCGCTTGTGCGGGCCCCCGTCAATTCCTTTGAGTTTTAGCCTTGCG GCCGTACTCCCCAGGCGGGGCGCTTAATGCGTTAGCTACGGCACGGGAACCGTGGAAA GCCCCCACACCTAGCGCCCAACGTTTACGGCGTGGACTACCAGGGTATCTAATCCTGTT CGCTCCCCACGCTTTCGCTCCTCAGCGTCAGGTAAGGCCCAGAGACCCGCCTTCGCCA CCGGTGTTCCTCCTGATATCTGCGCATTTCACCGCTACACCAGGAATTCCAGTCTCCCCT ACCTACCTCTAGCATGCCCGTATCCACTGCAAAACCAGGGTTAAGCCCCAGCCTTTCAC AGCAGACGCGACACACCGCCTACGAGCTCTTTACGCCCAATAATTCCGGACAACGCTC GGACCCTACGTATTACCGCGGCTGCTGGCACGTAGTTAGCCGGTCCTTATTCCCCACCT ACCGTCAACCCCGAGAACACCCGGGGCCTGCGTGGAGTGGTAAAAGAGGTTTACAAC CCGAAGGCGTCATCCCCCCACGCGGCGTCGCTGCGTCAGGCTTTCGTCCATGCGCAGA TCCTCACTGCTGCTTCCCGCAGAGTCTGGTCGTGTCTCAGTTCCAGTGTGTCGTTCGCC CTCTCAGCCGGCTACGTATCGACTTGGTAGCCTACCCCACCAACAAGCTGATATAGGAC CGCGCGGAG 
Figure S3. Detailed molecular network of Nocardiopsis sp. HDN154086.

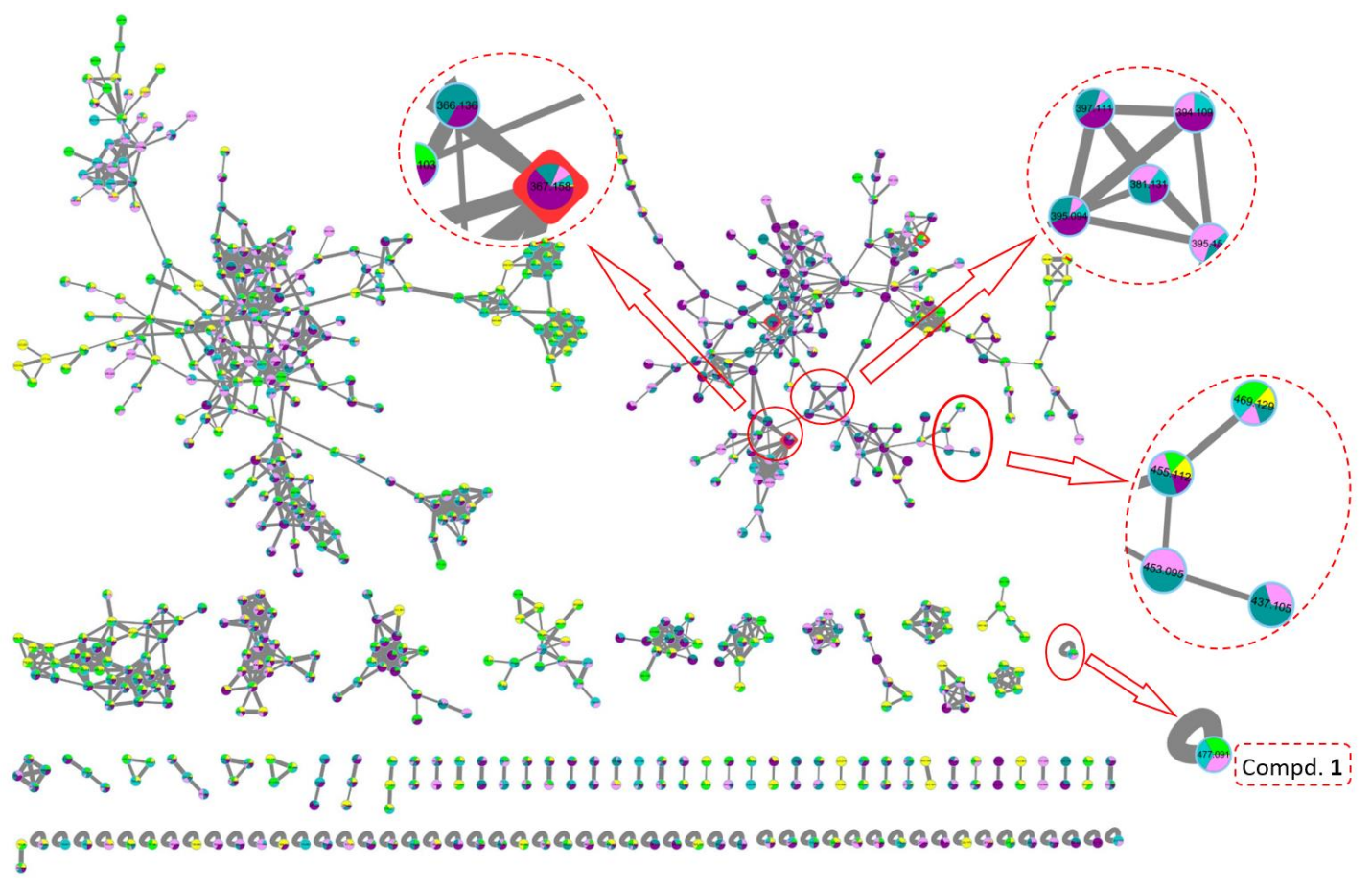

Figure S4. The MS/MS spectra of the compounds.
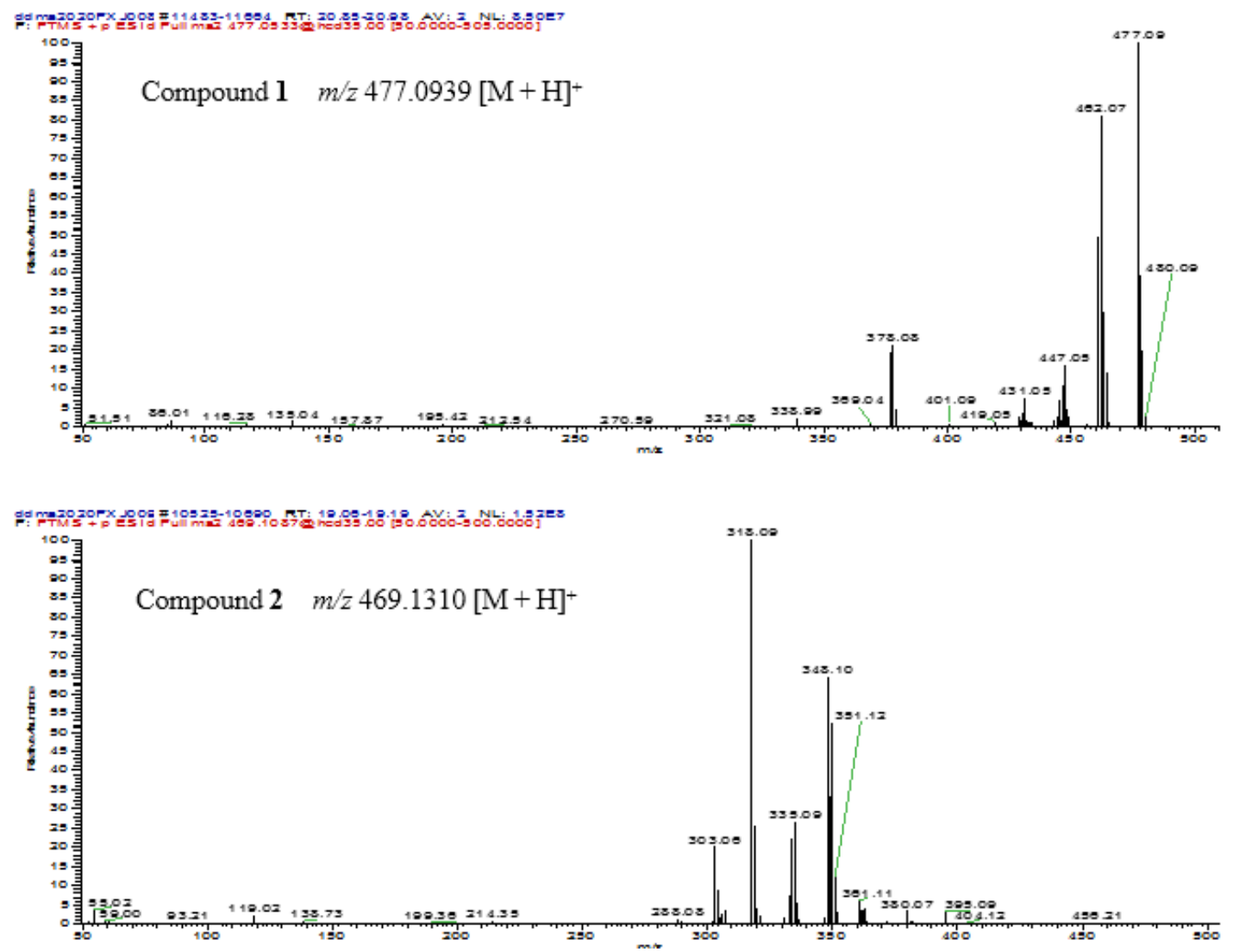

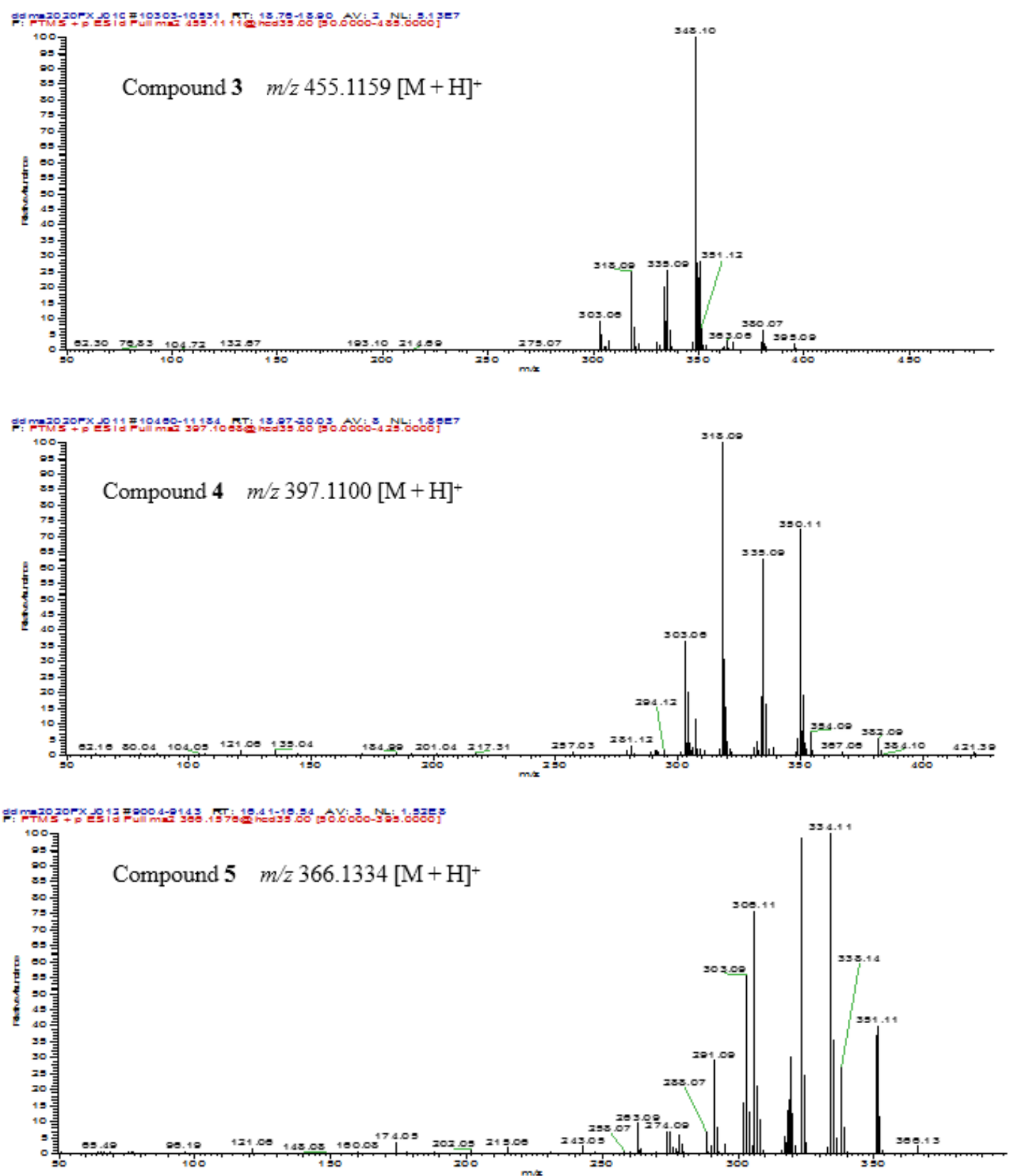
Figure S5. ${ }^{1} \mathrm{H}$ NMR $\left(500 \mathrm{MHz}, \mathrm{CDCl}_{3}\right)$ spectrum of nocarterphenyl D (1).

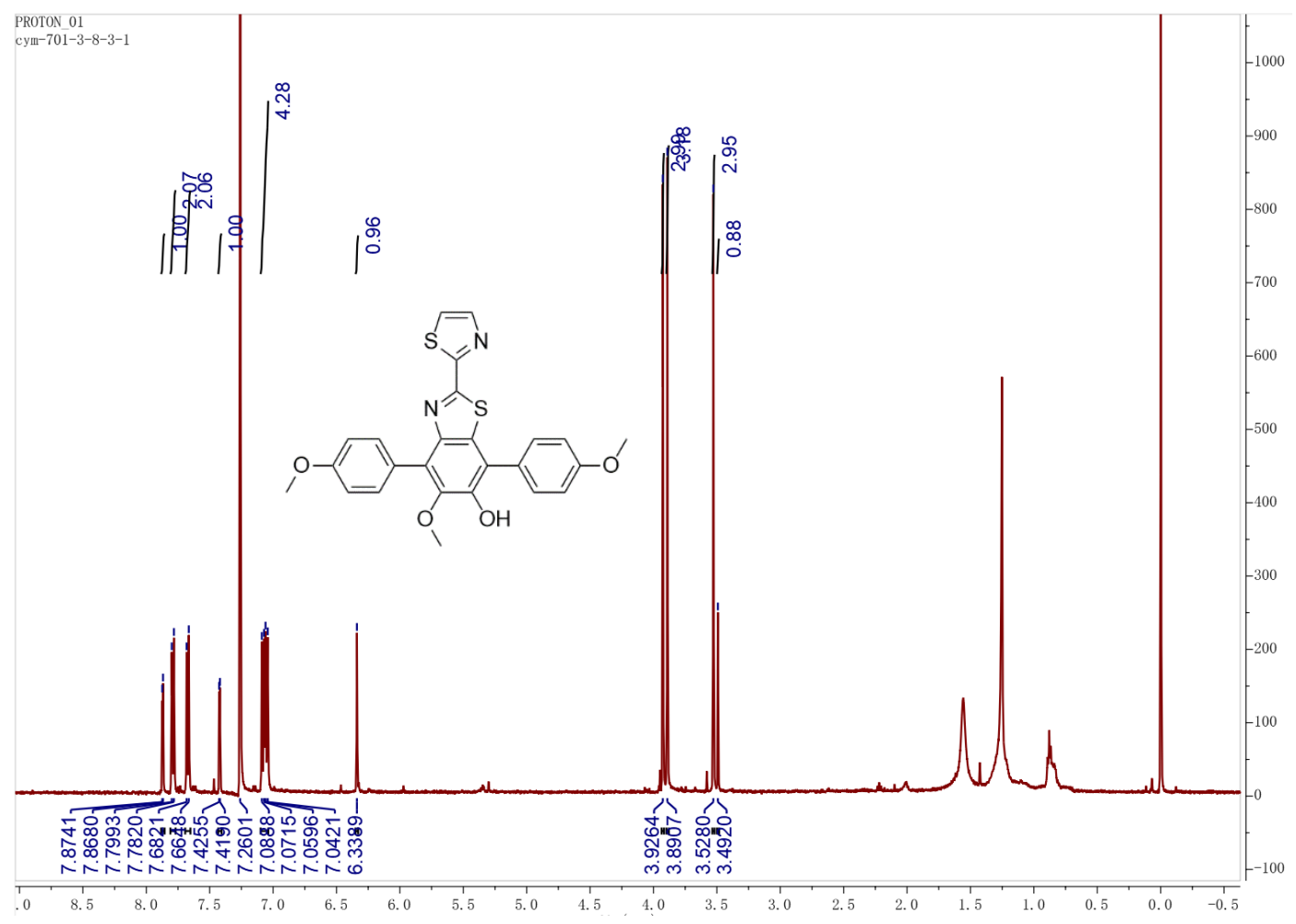

Figure S6. ${ }^{13} \mathrm{C}$ NMR $\left(125 \mathrm{MHz}, \mathrm{CDCl}_{3}\right)$ spectrum of nocarterphenyl D (1).

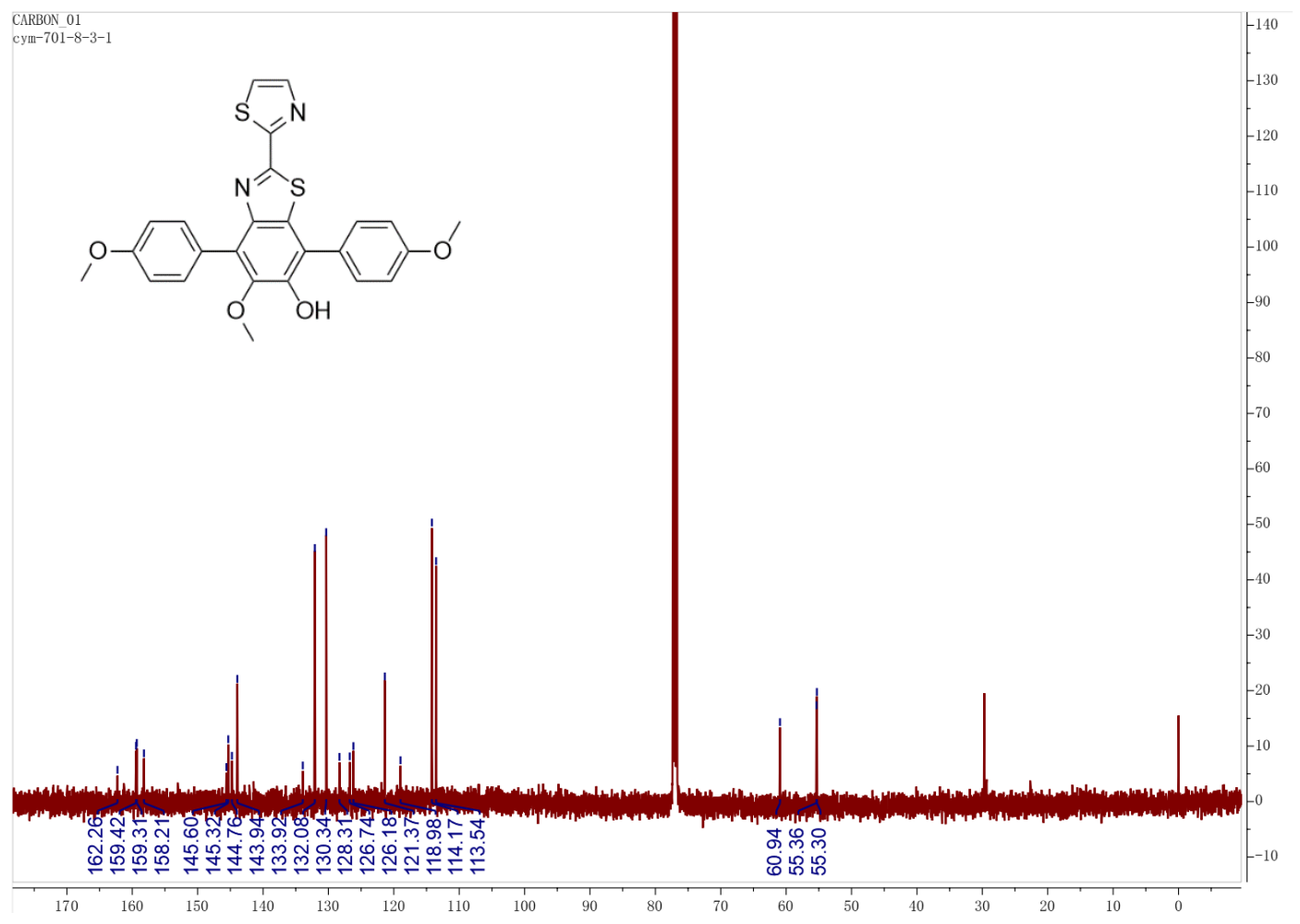


Figure S7. DEPT (125 MHz, $\left.\mathrm{CDCl}_{3}\right)$ spectrum of nocarterphenyl D (1).
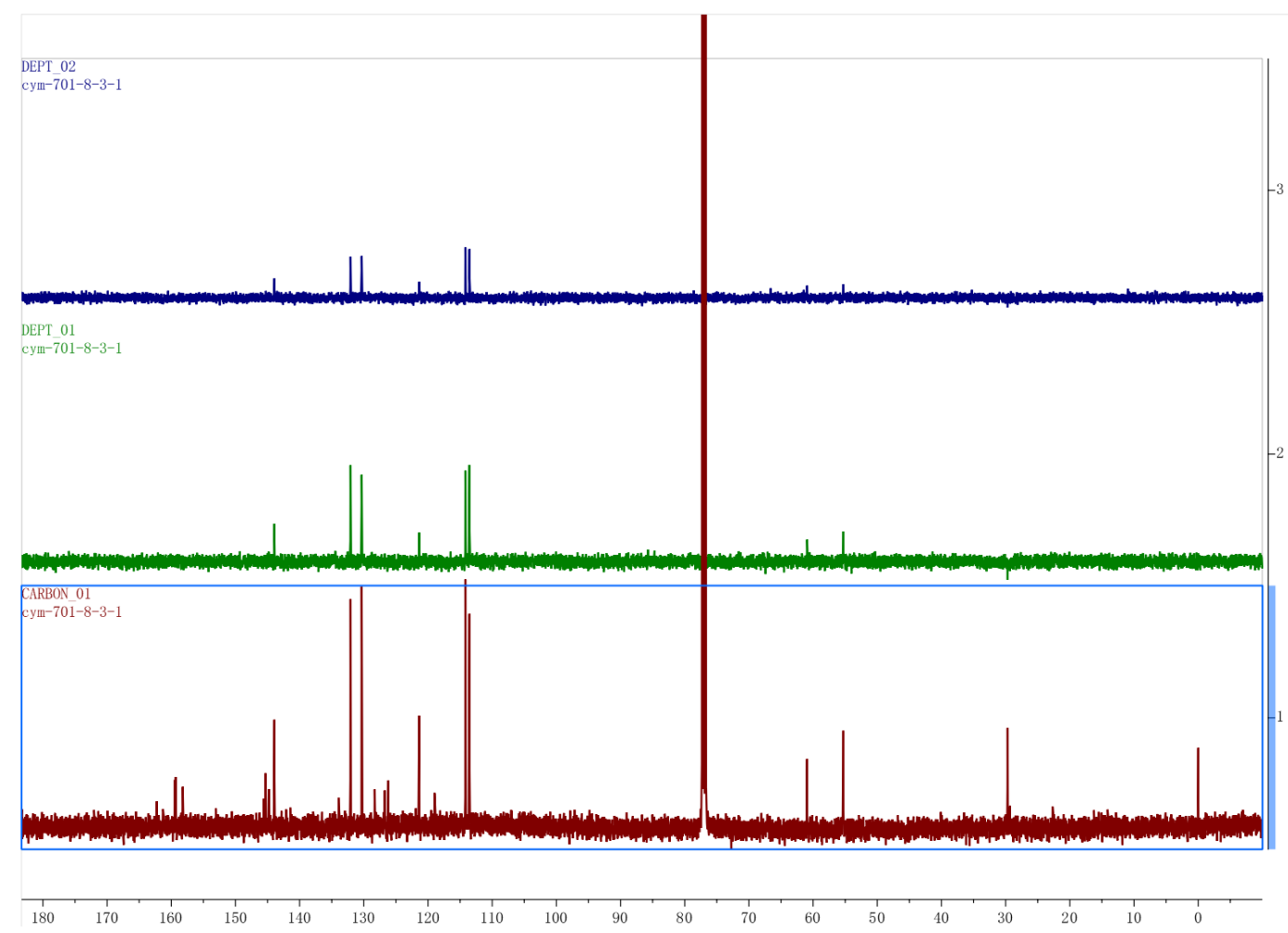

Figure S8. HSQC spectrum of nocarterphenyl D (1).

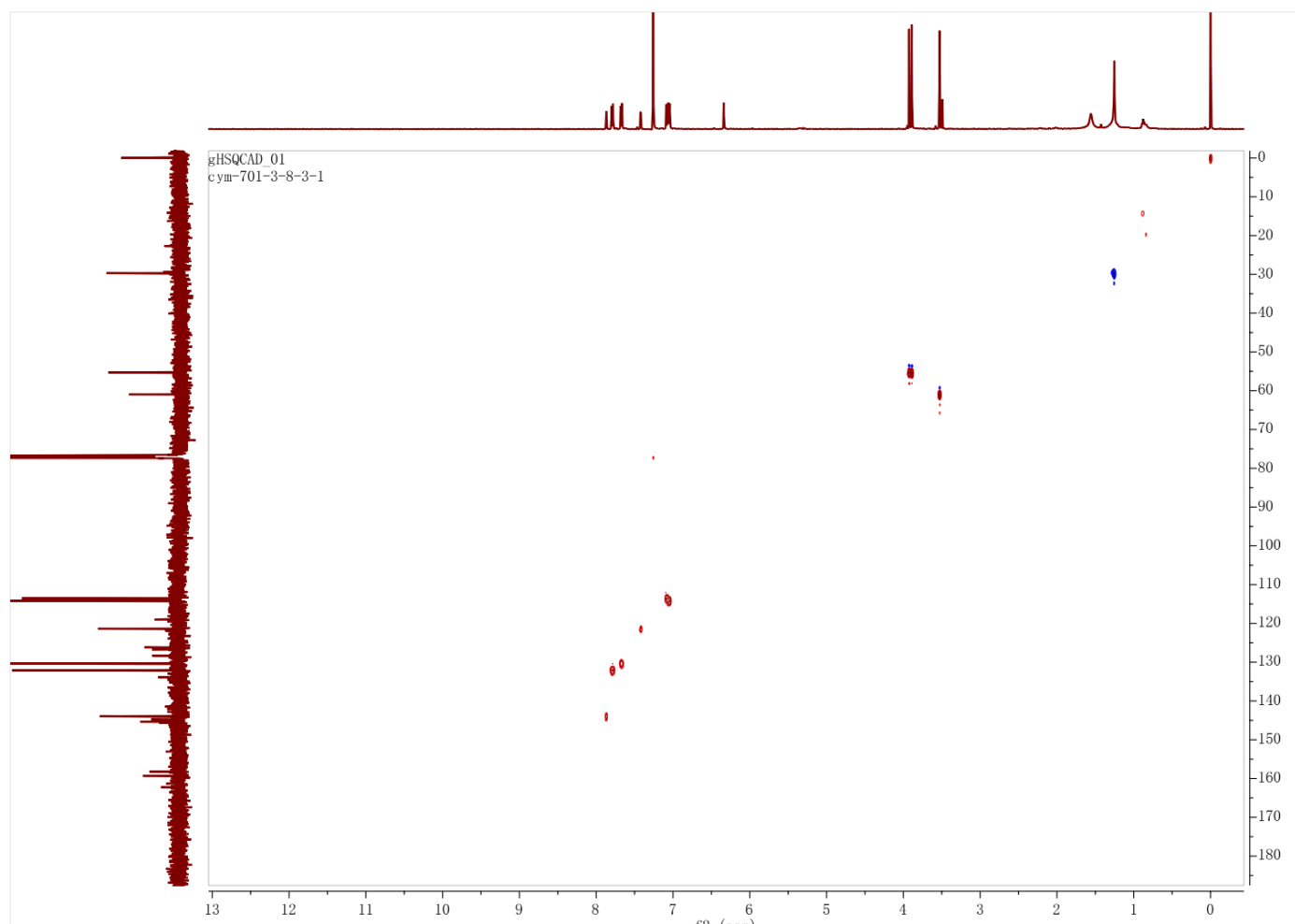


Figure S9. ${ }^{1} \mathrm{H}-{ }^{1} \mathrm{H}$ COSY spectrum of nocarterphenyl D (1).

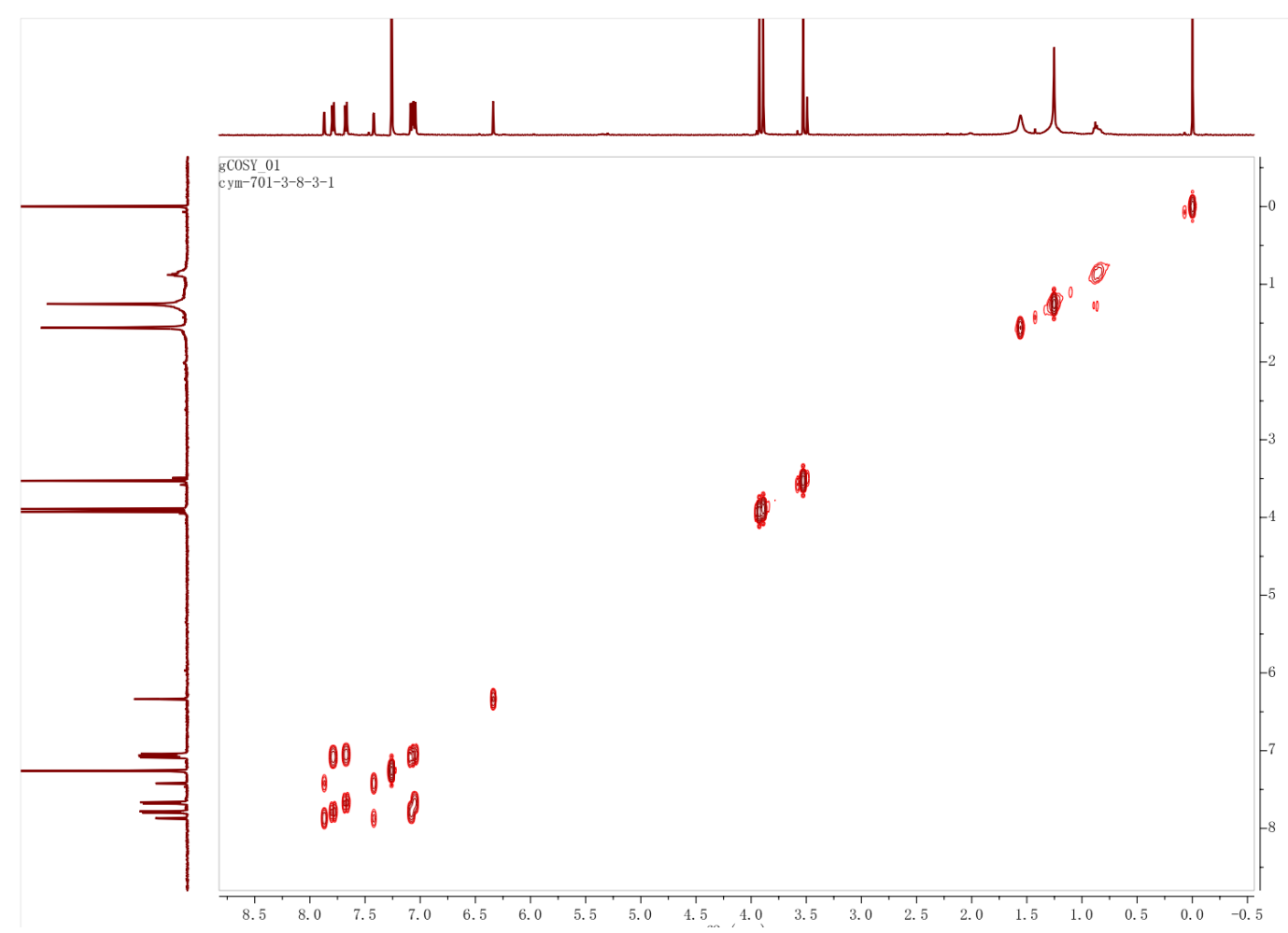

Figure S10. ${ }^{1} \mathrm{H}^{-13} \mathrm{C}$ HMBC spectrum of nocarterphenyl D (1).

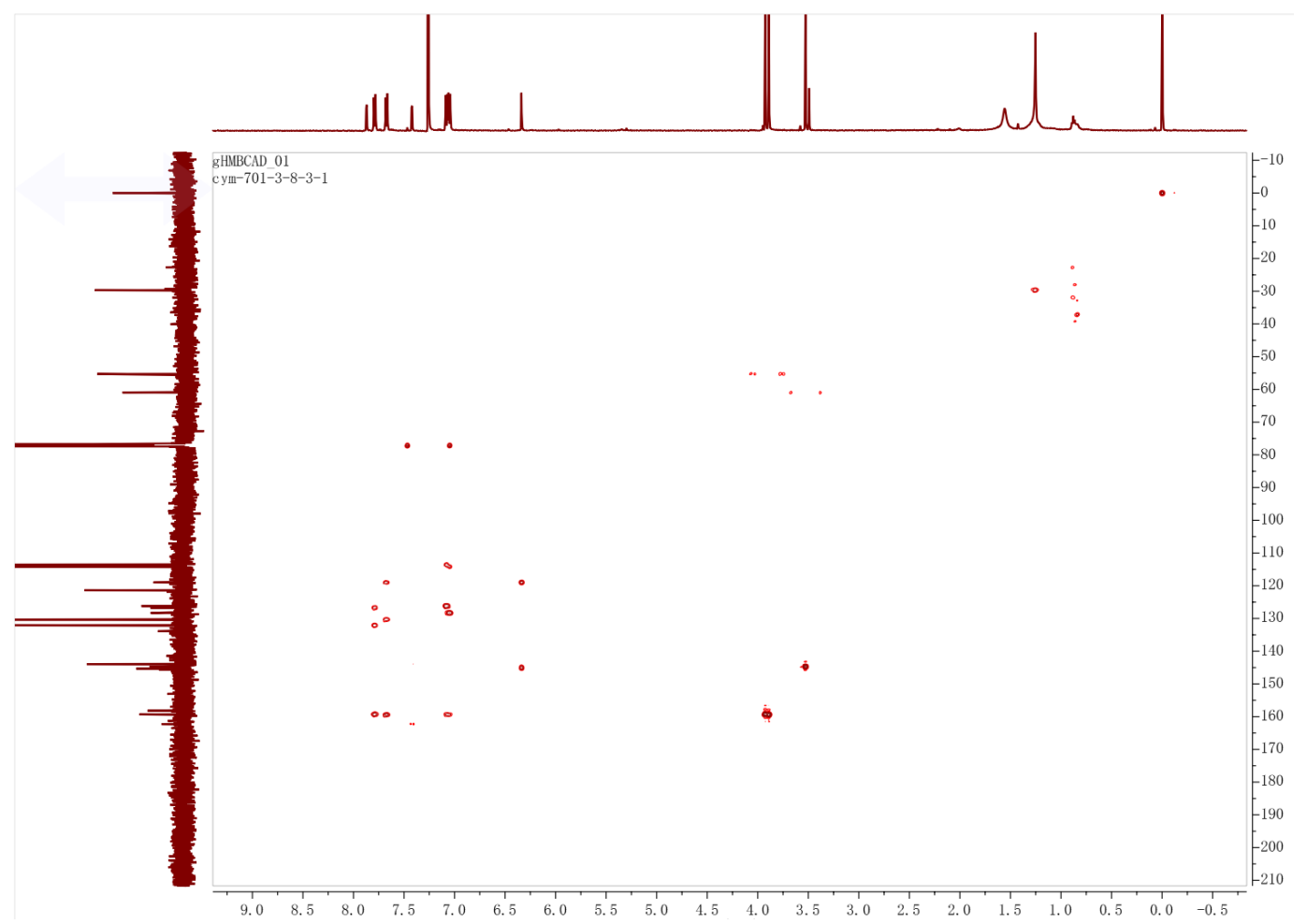


Figure S11. HRESIMS spectrum of nocarterphenyl D (1).

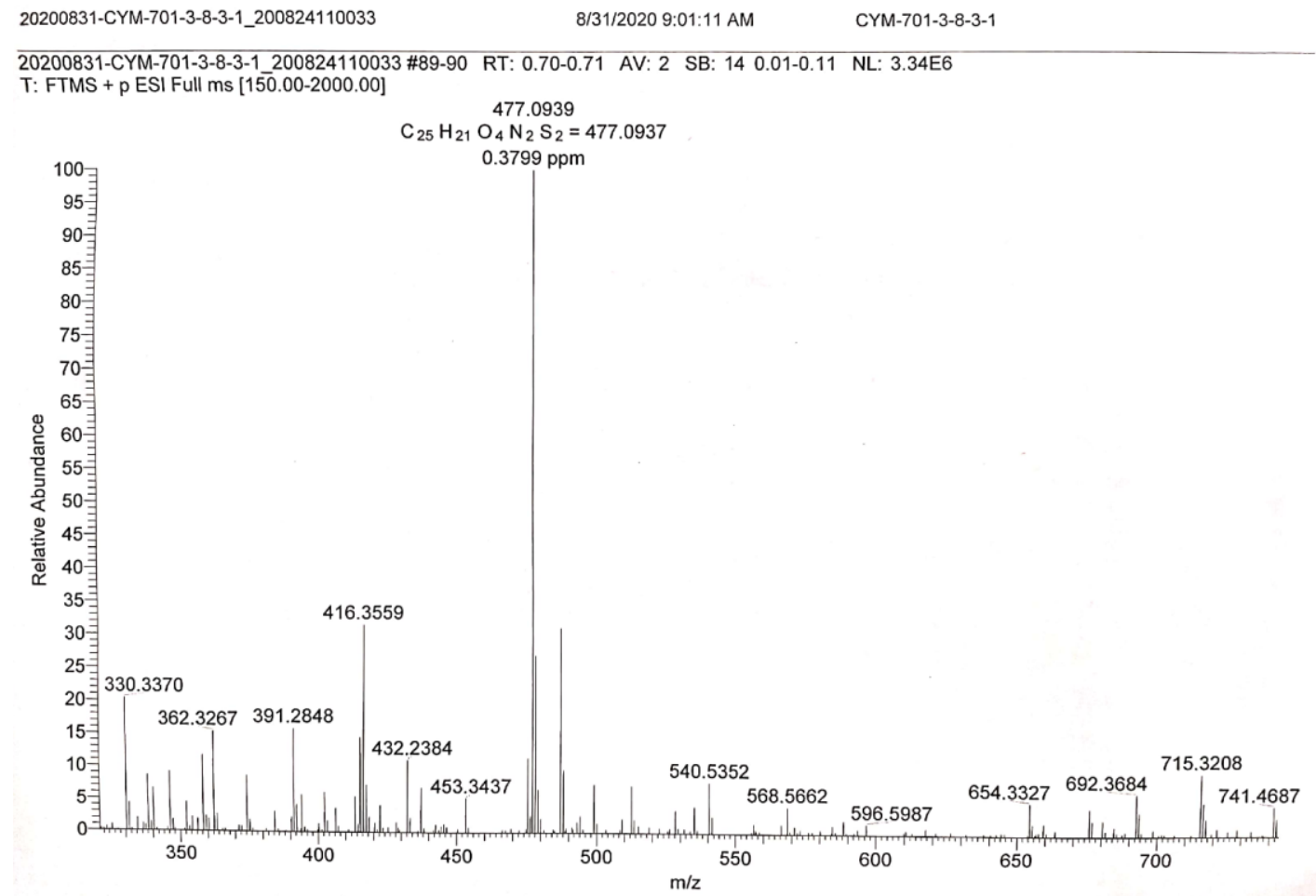

Figure S12. IR spectrum of nocarterphenyl D (1).

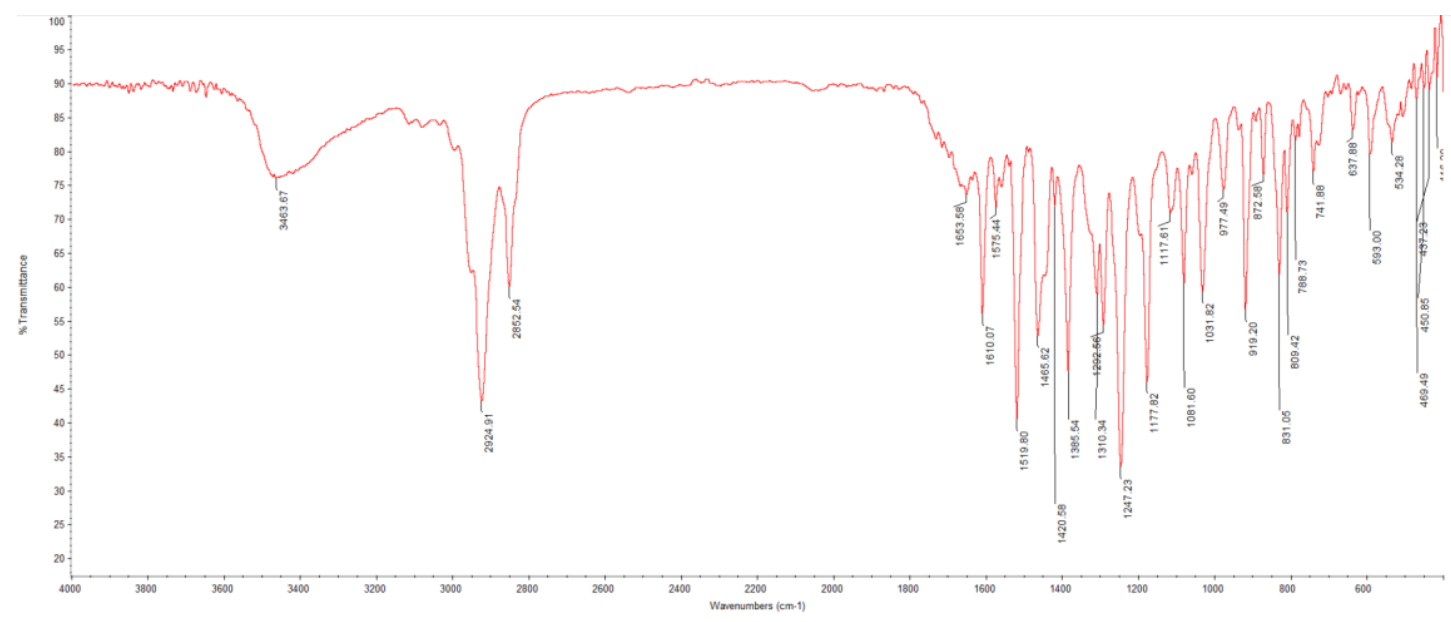


Figure S13. ${ }^{1} \mathrm{H}$ NMR $\left(500 \mathrm{MHz}, \mathrm{CDCl}_{3}\right)$ spectrum of nocarterphenyl E (2).

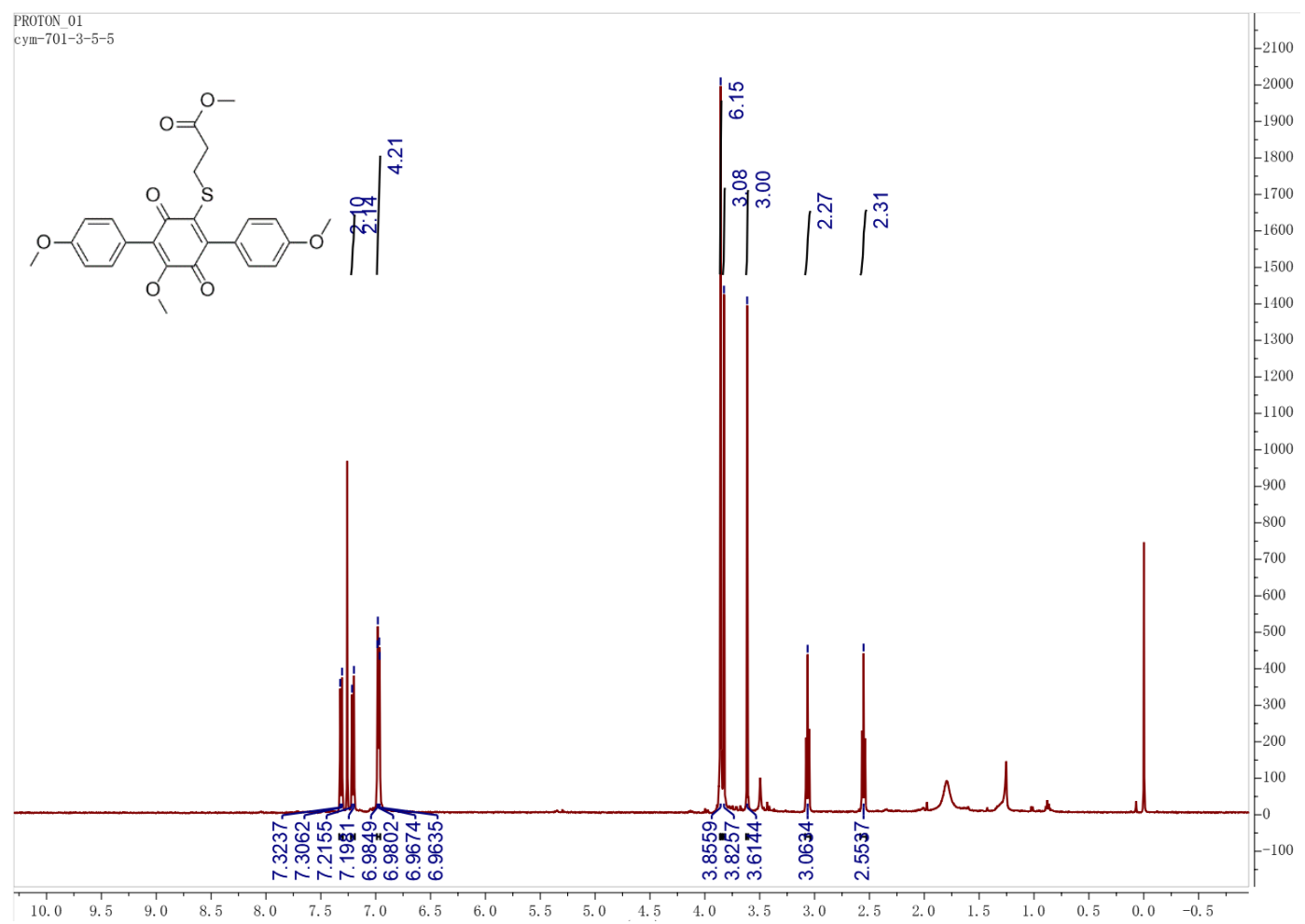

Figure S14. ${ }^{13} \mathrm{C}$ NMR $\left(150 \mathrm{MHz}, \mathrm{CDCl}_{3}\right)$ spectrum of nocarterphenyl E (2).

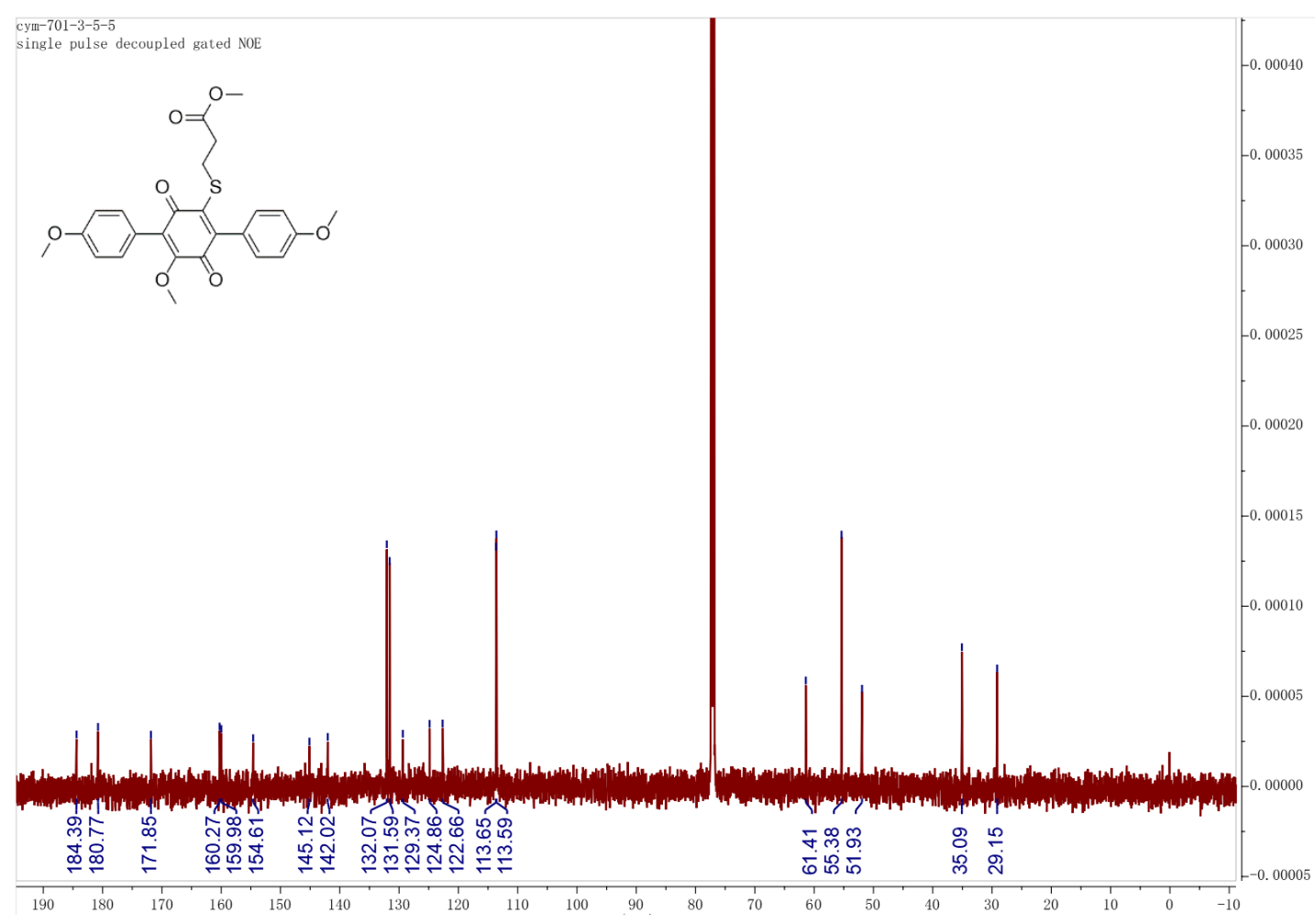


Figure S15. DEPT (150 MHz, $\mathrm{CDCl}_{3}$ ) spectrum of nocarterphenyl E (2).

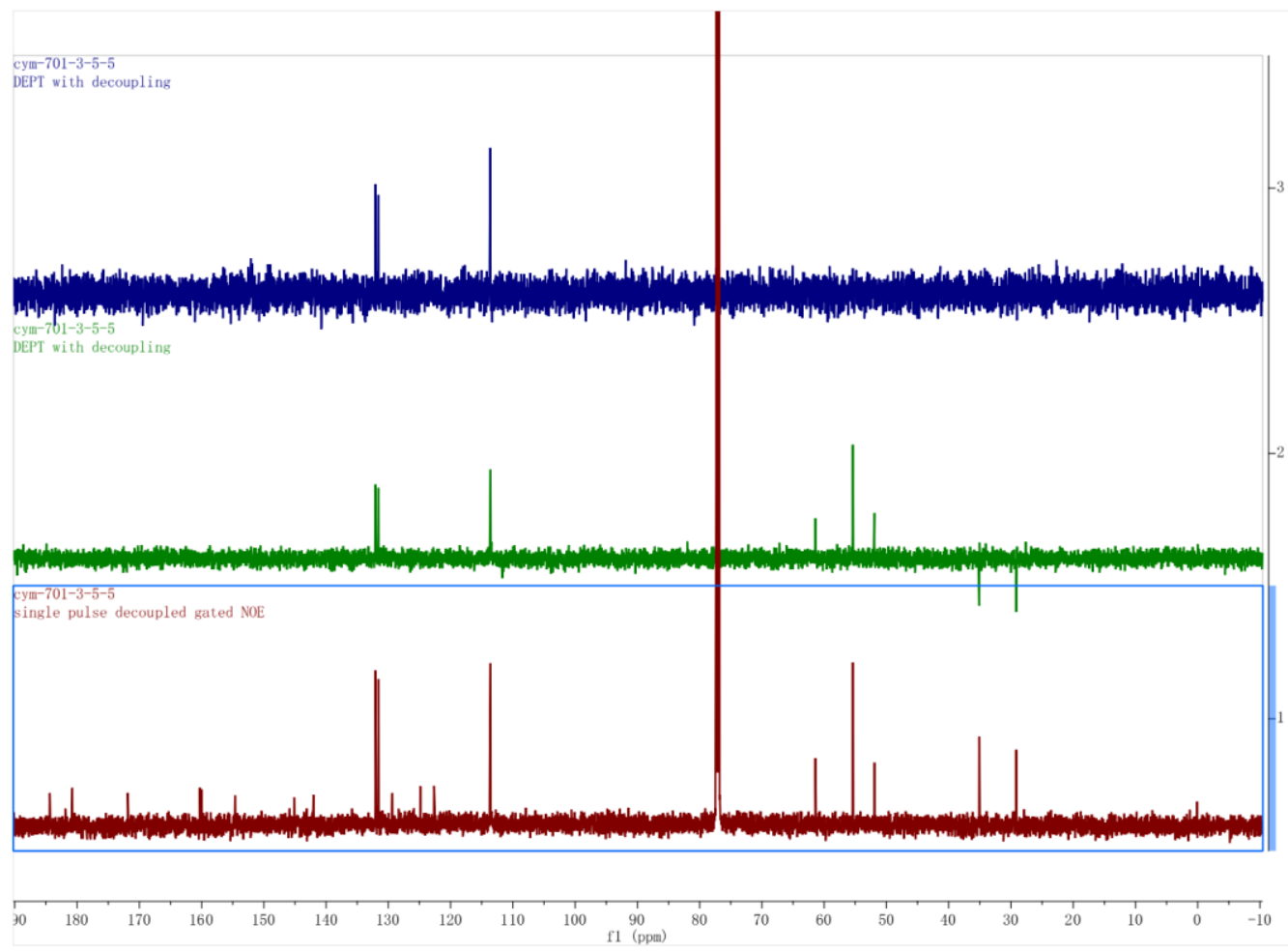

Figure S16. HSQC spectrum of nocarterphenyl E (2).

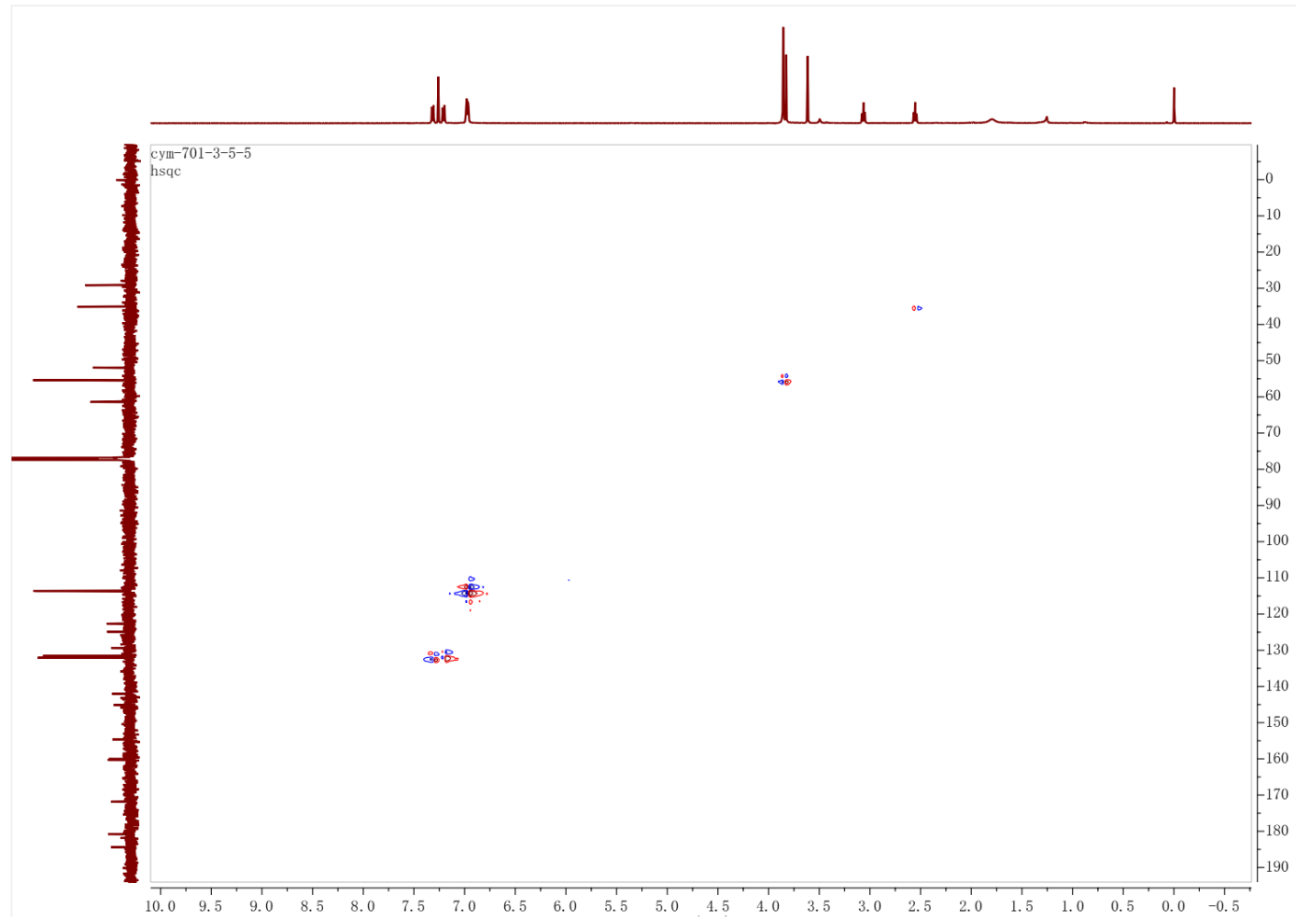


Figure S17. ${ }^{1} \mathrm{H}-{ }^{1} \mathrm{H}$ COSY spectrum of nocarterphenyl E (2).

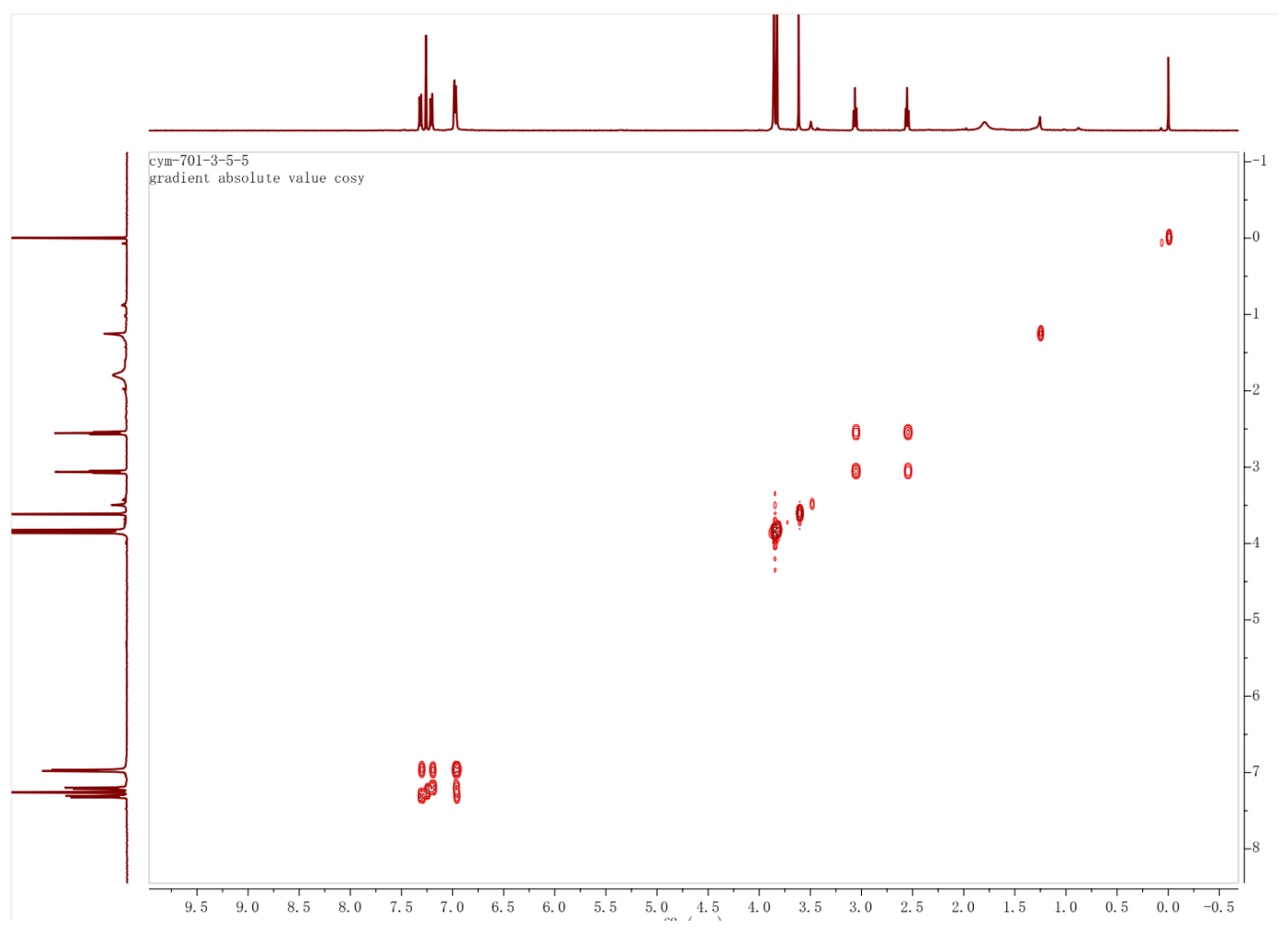

Figure S18. ${ }^{1} \mathrm{H}-{ }^{13} \mathrm{C}$ HMBC spectrum of nocarterphenyl E (2).

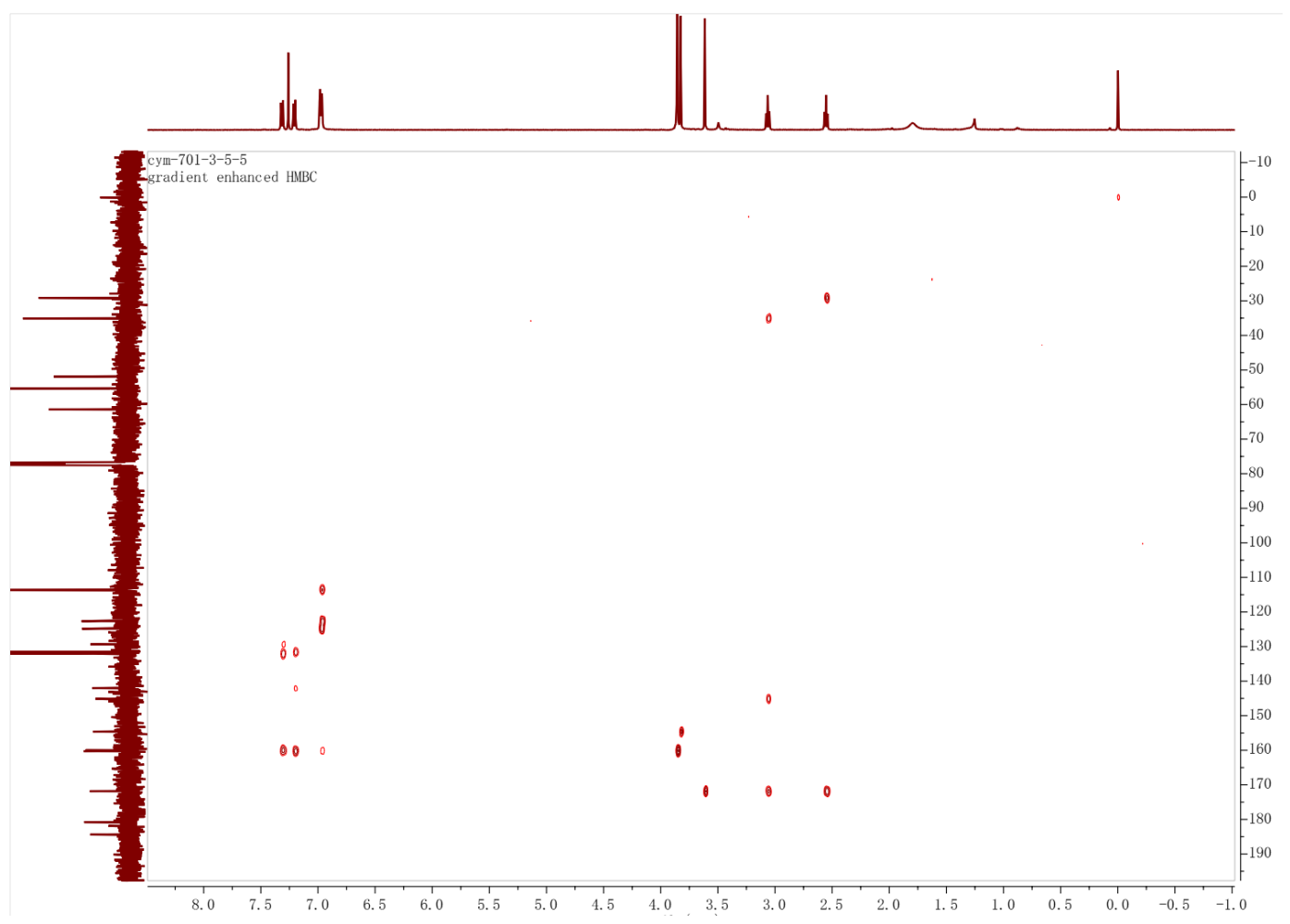

13 
Figure S19. HRESIMS spectrum of nocarterphenyl E (2).

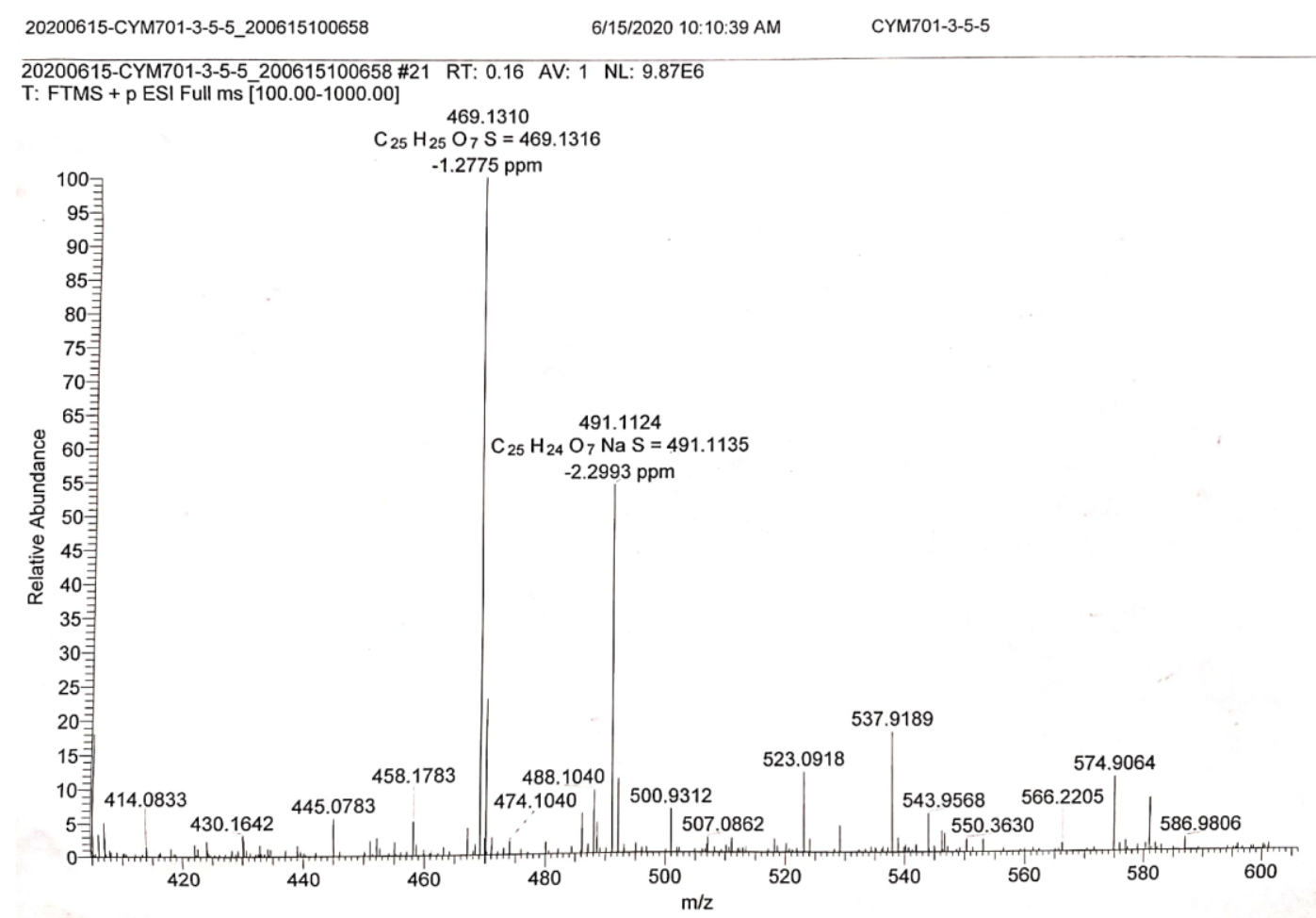

Figure S20. IR spectrum of nocarterphenyl E (2).

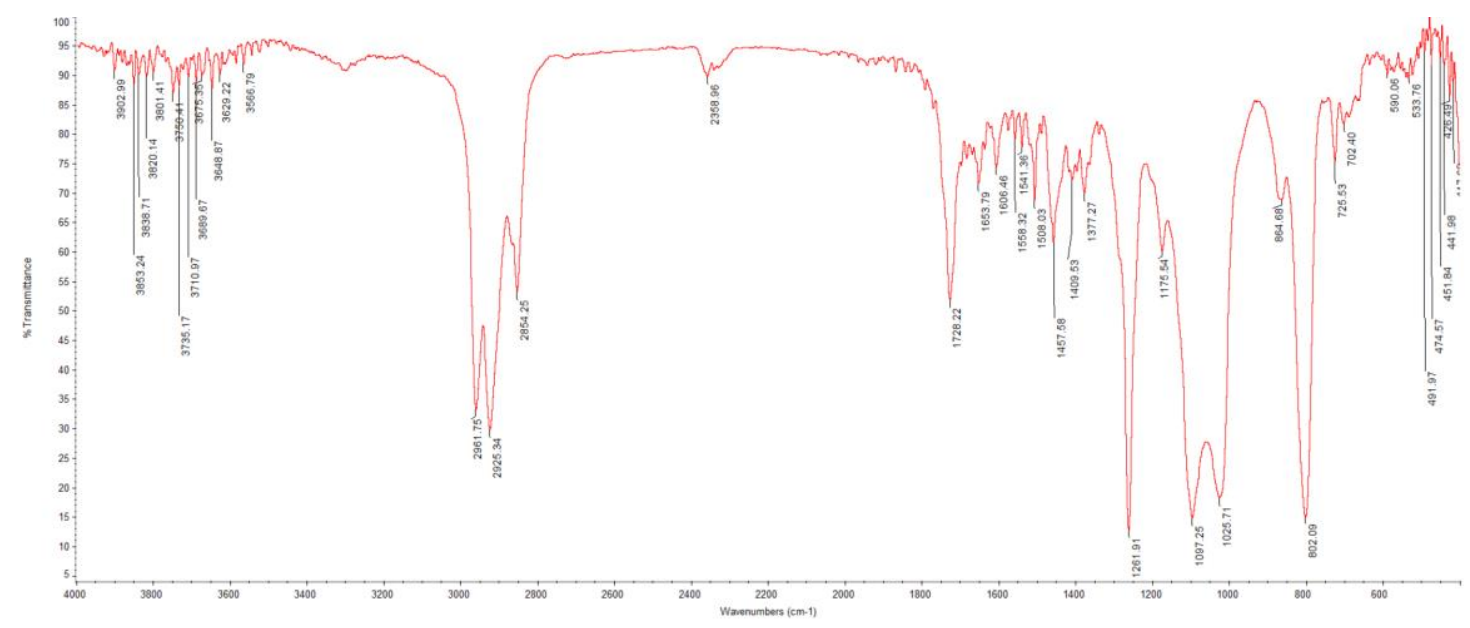


Figure S21. ${ }^{1} \mathrm{H}$ NMR (600 MHz, DMSO) spectrum of nocarterphenyl F (3).

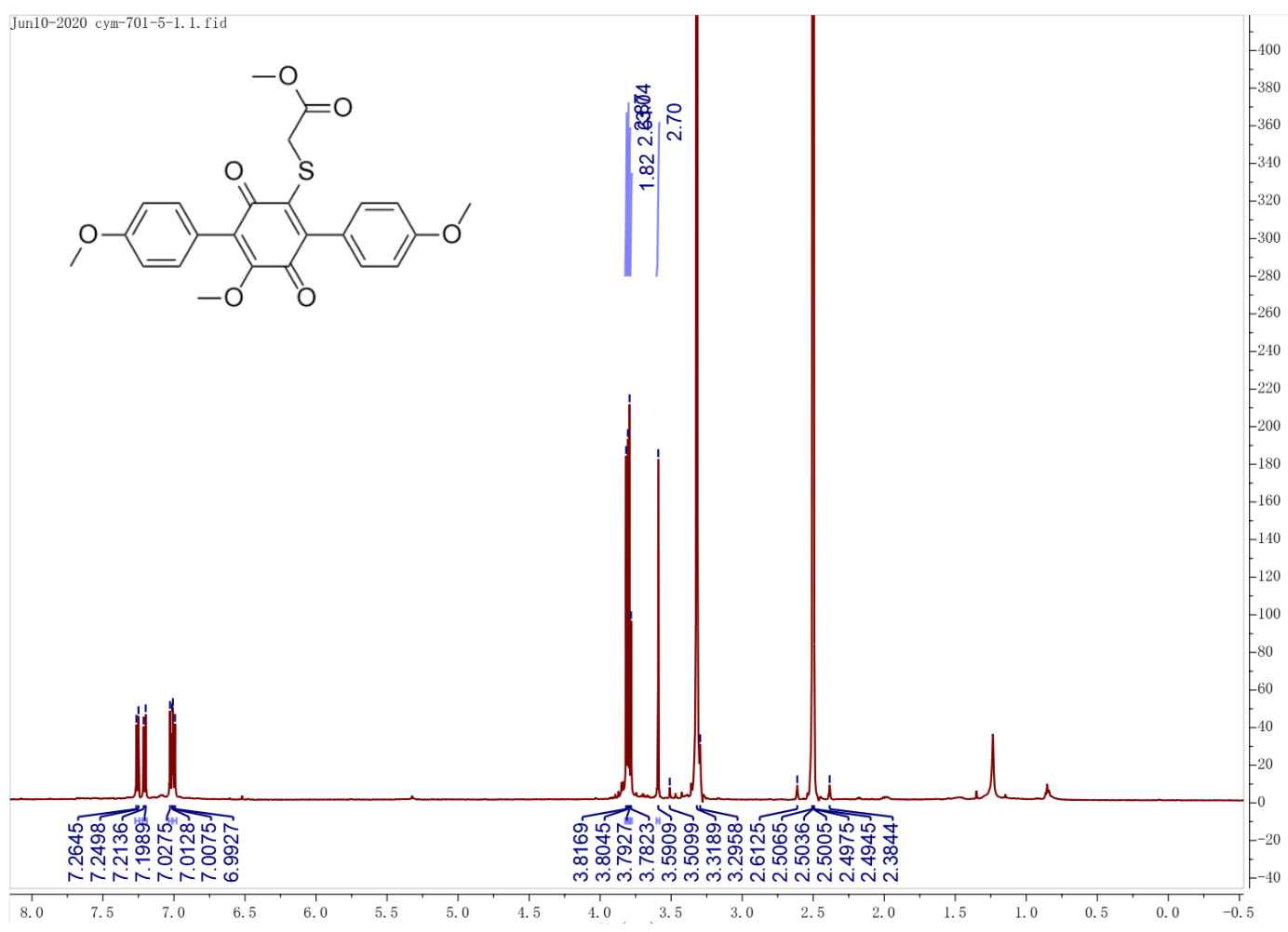

Figure S22. ${ }^{13} \mathrm{C}$ NMR (150 MHz, DMSO) spectrum of nocarterphenyl F (3).

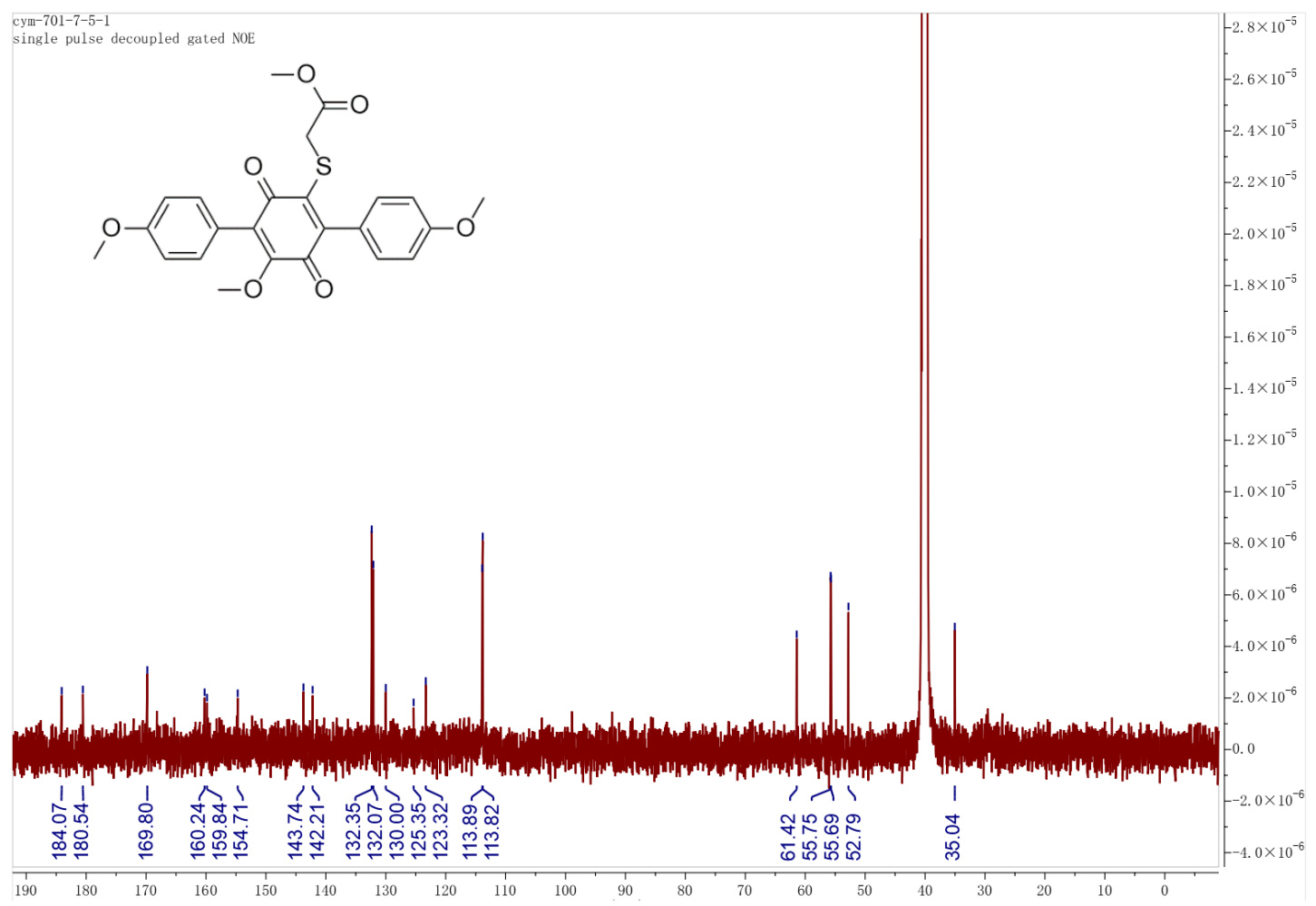


Figure S23. DEPTQ (150 MHz, $\left.\mathrm{CDCl}_{3}\right)$ spectrum of nocarterphenyl $\mathrm{F}(3)$.

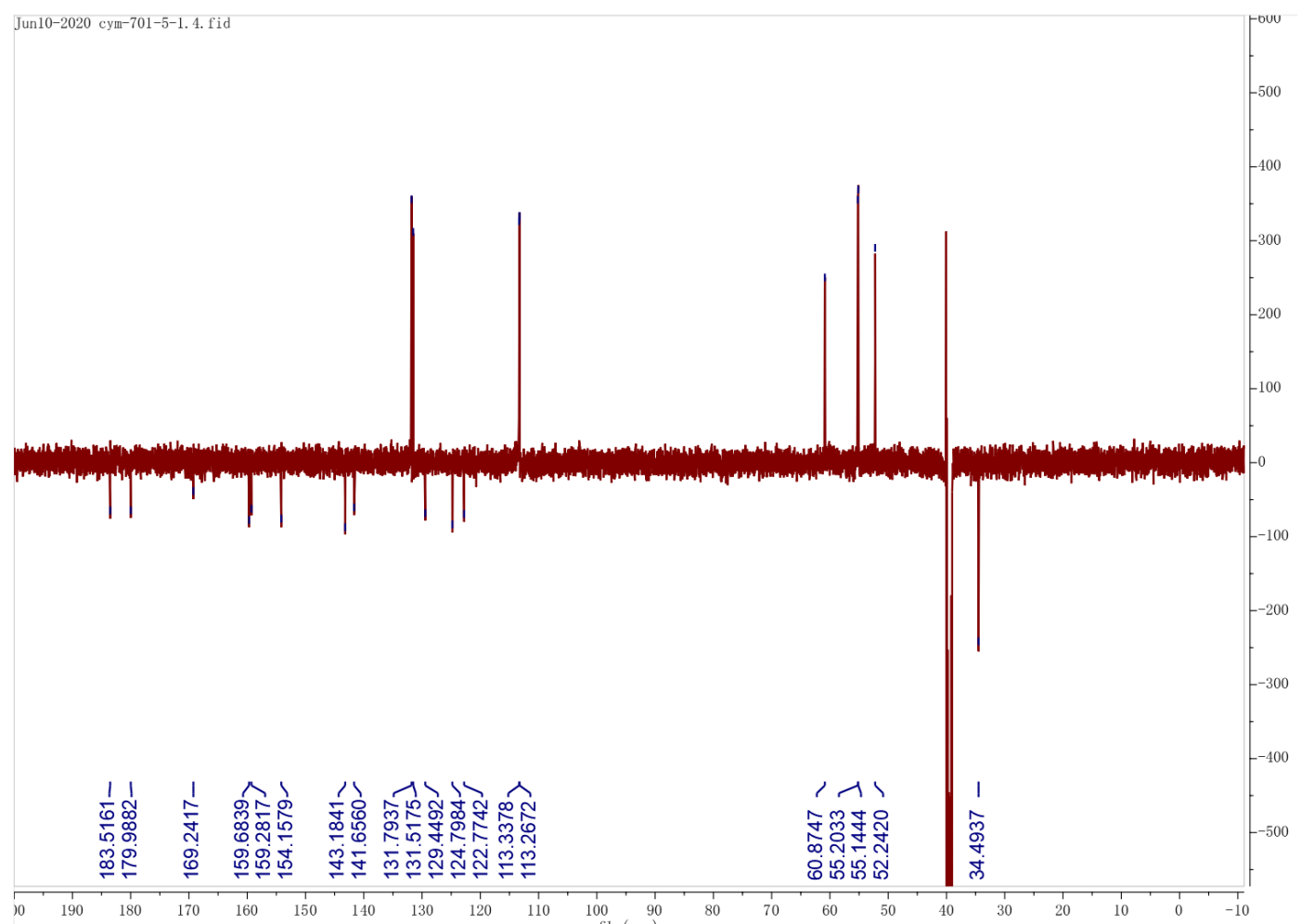

Figure S24. HSQC spectrum of nocarterphenyl F (3).

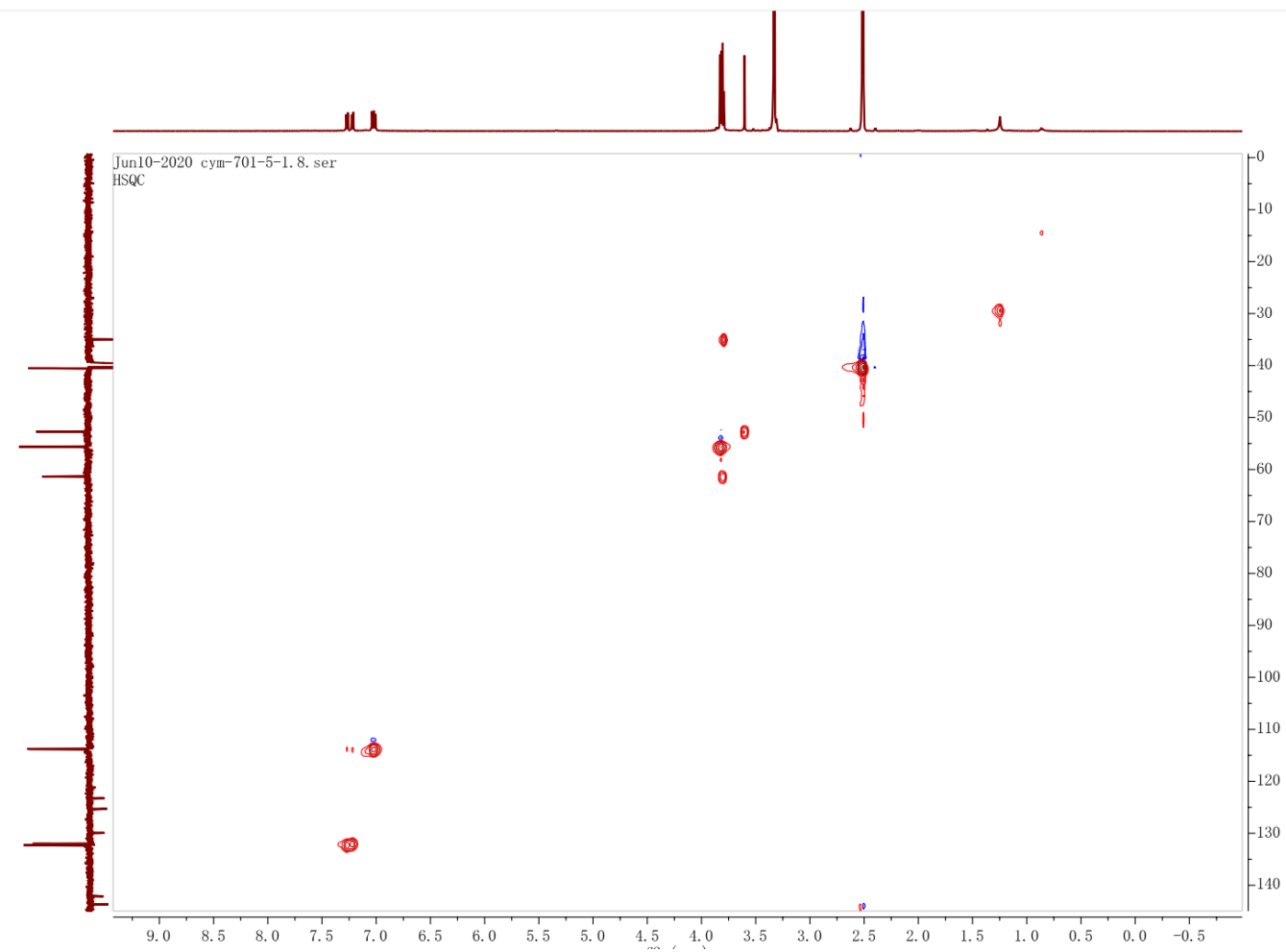


Figure S25. ${ }^{1} \mathrm{H}-{ }^{1} \mathrm{H}$ COSY spectrum of nocarterphenyl F (3).

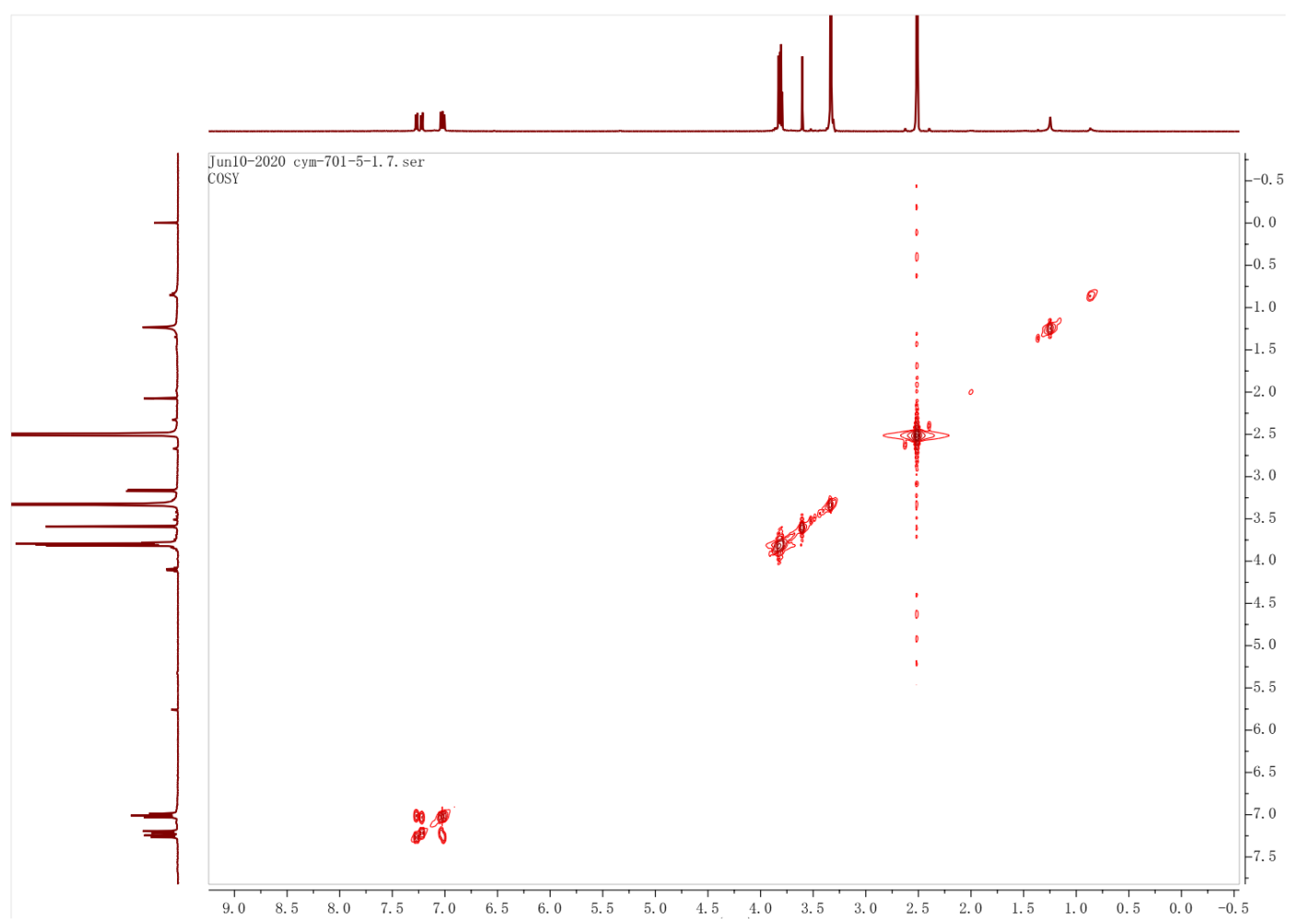

Figure S26. ${ }^{1} \mathrm{H}^{-13} \mathrm{C}$ HMBC spectrum of nocarterphenyl $\mathrm{F}(3)$.

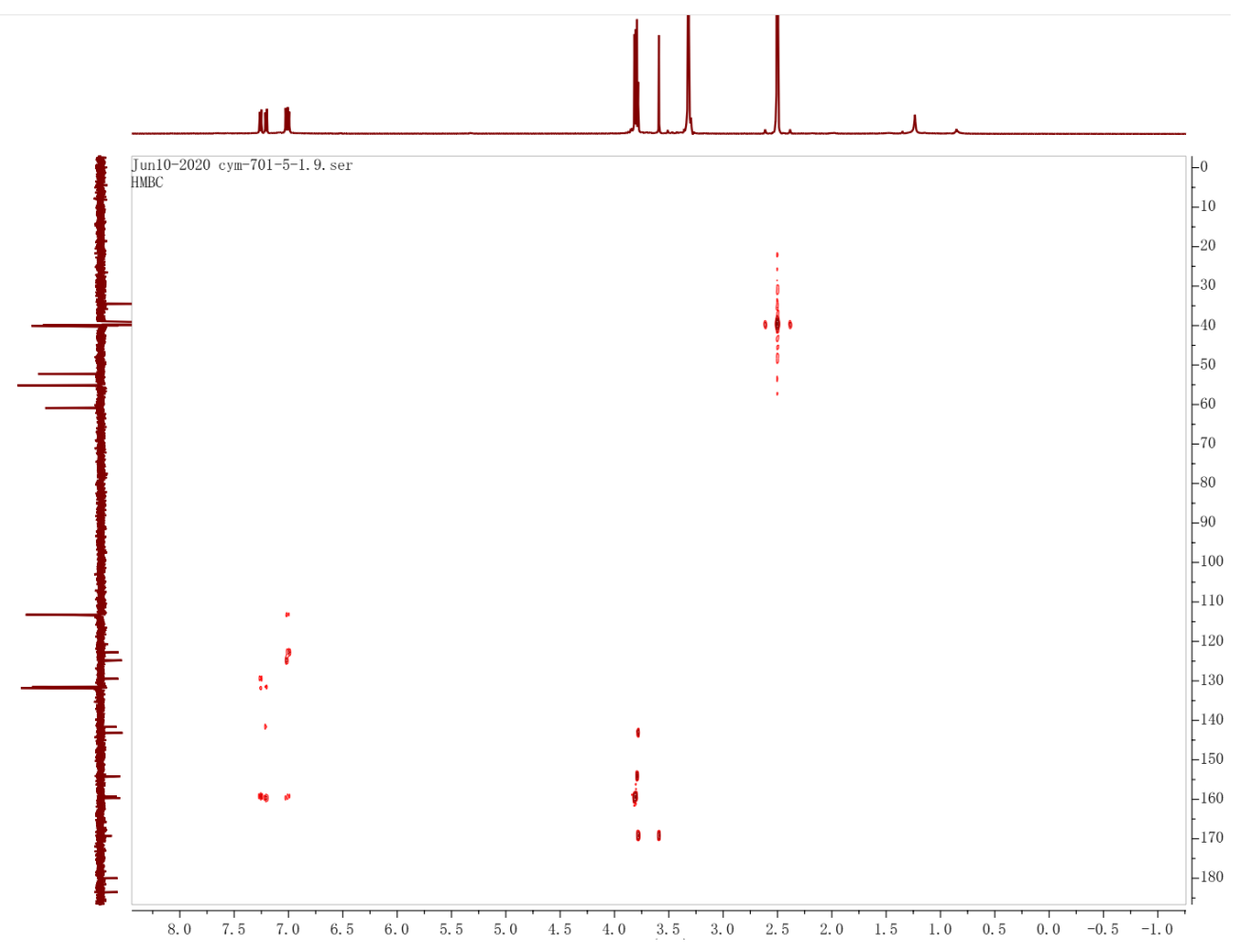


Figure S27. HRESIMS spectrum of nocarterphenyl F (3).

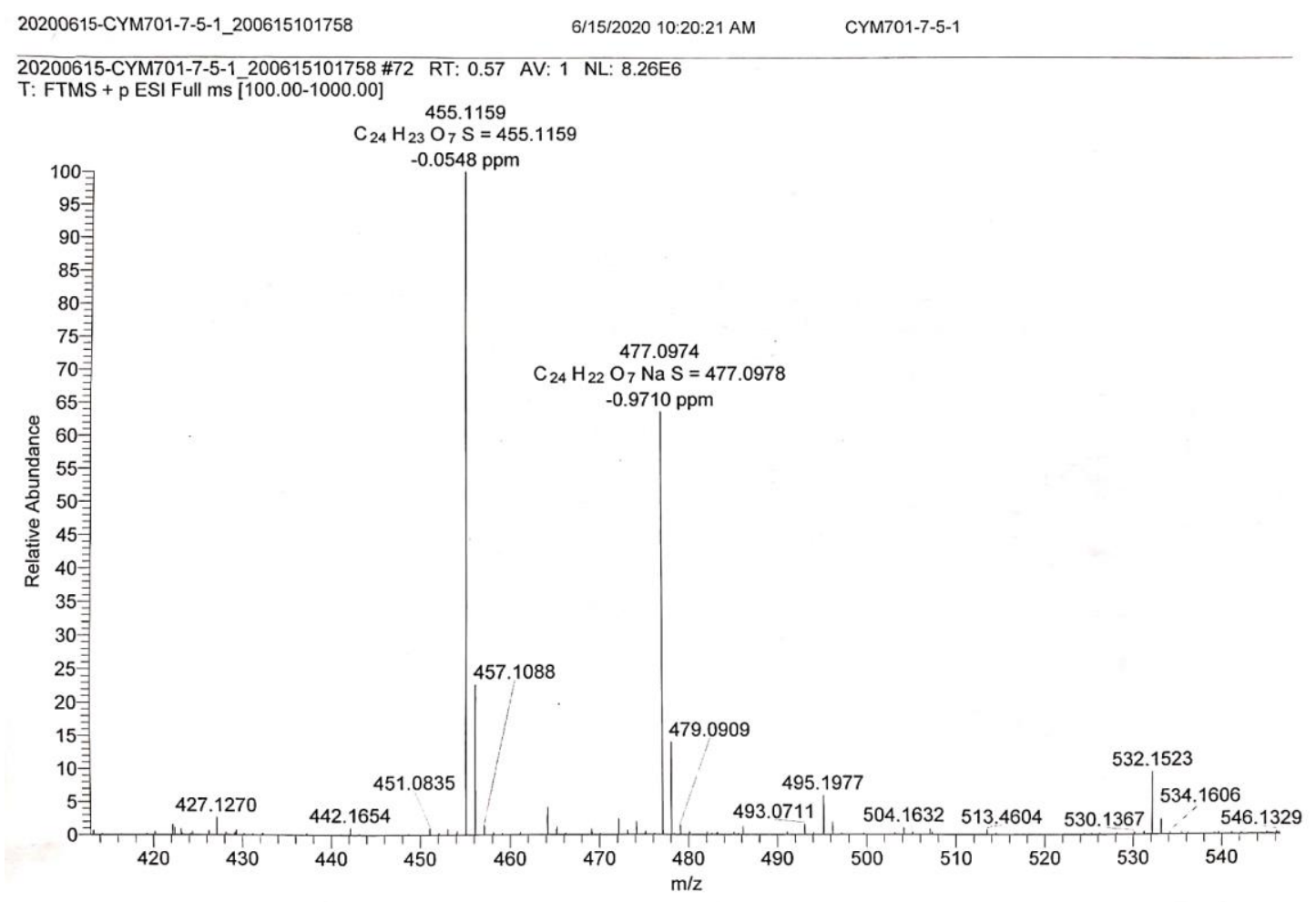

Figure S28. IR spectrum of nocarterphenyl F (3).

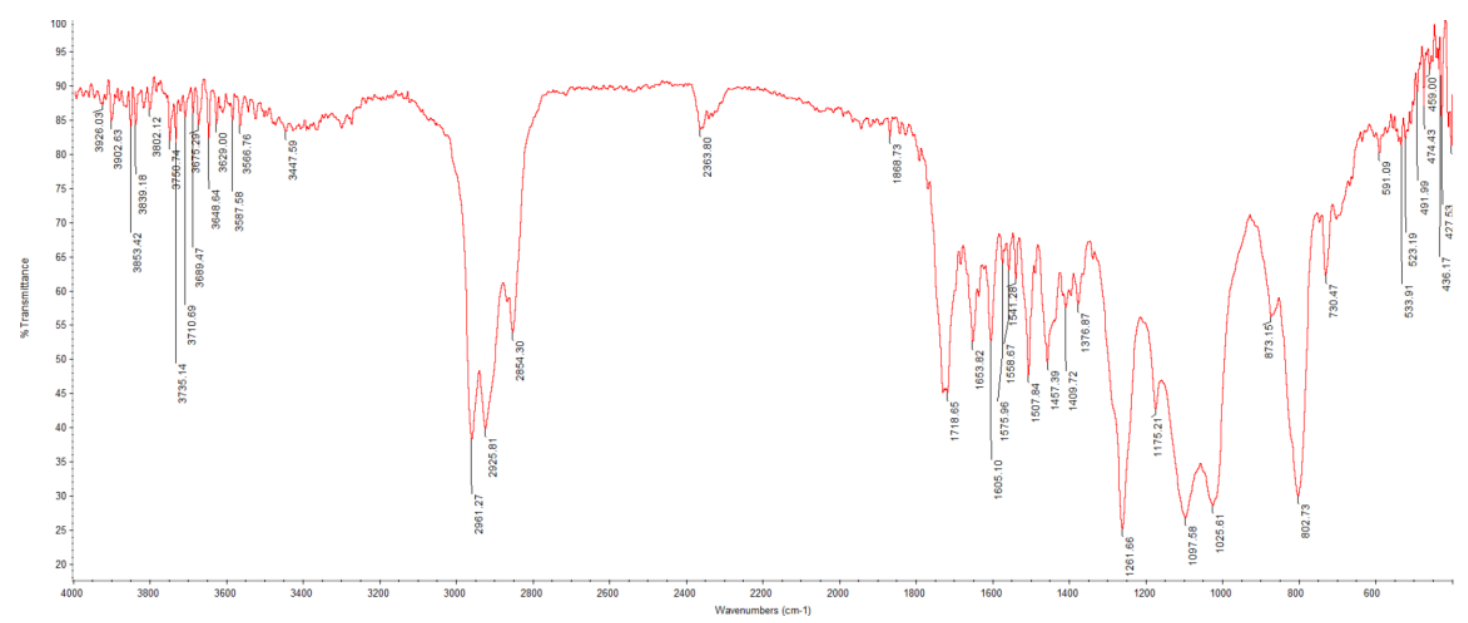


Figure S29. ${ }^{1} \mathrm{H}$ NMR (600 MHz, $\left.\mathrm{CDCl}_{3}\right)$ spectrum of nocarterphenyl G (4).

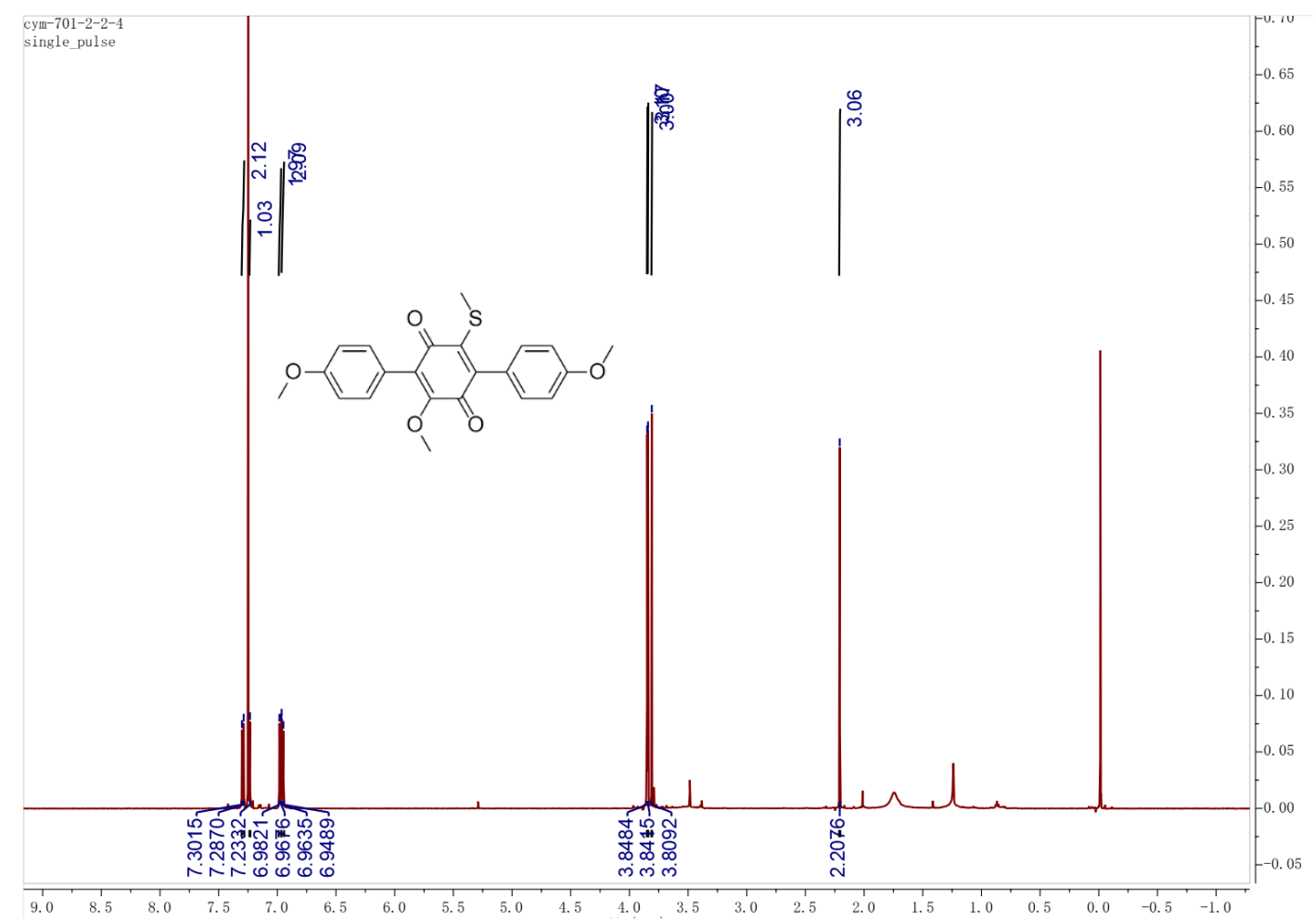

Figure S30. ${ }^{13} \mathrm{C}$ NMR (150 MHz, $\left.\mathrm{CDCl}_{3}\right)$ spectrum of nocarterphenyl G (4).

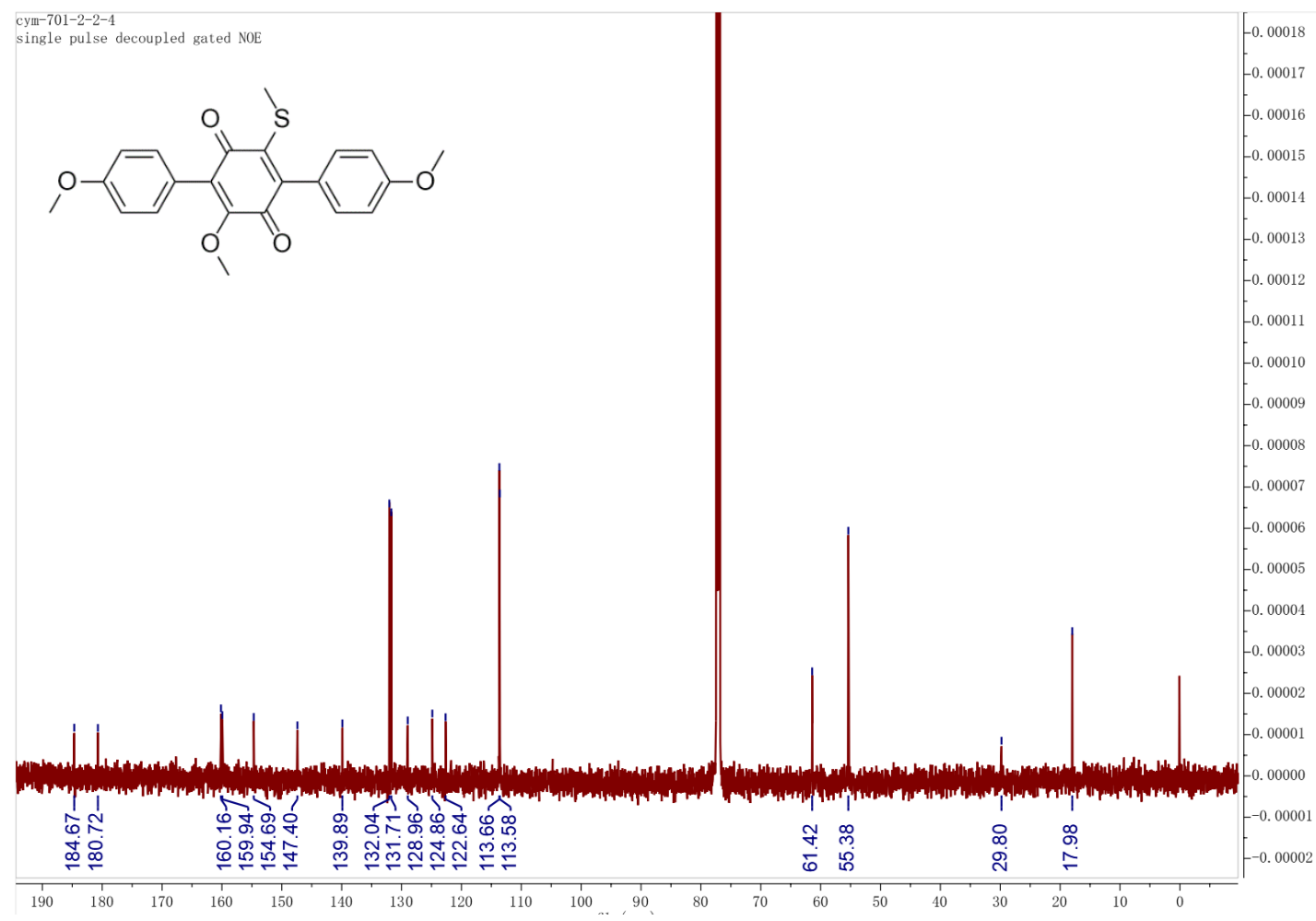


Figure S31. DEPT (150 MHz, $\mathrm{CDCl}_{3}$ ) spectrum of nocarterphenyl G (4).

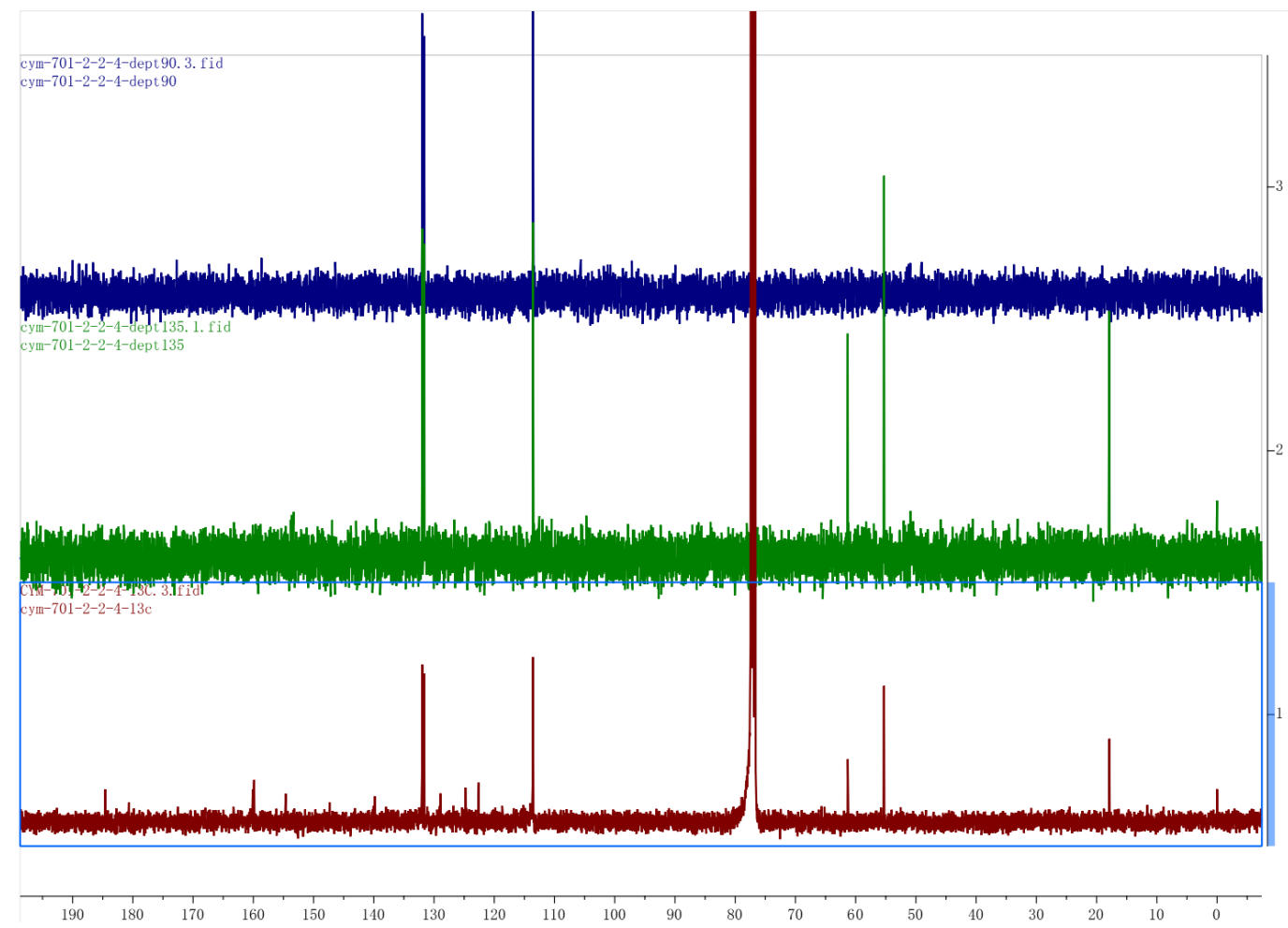

Figure S32. HSQC spectrum of nocarterphenyl G (4).

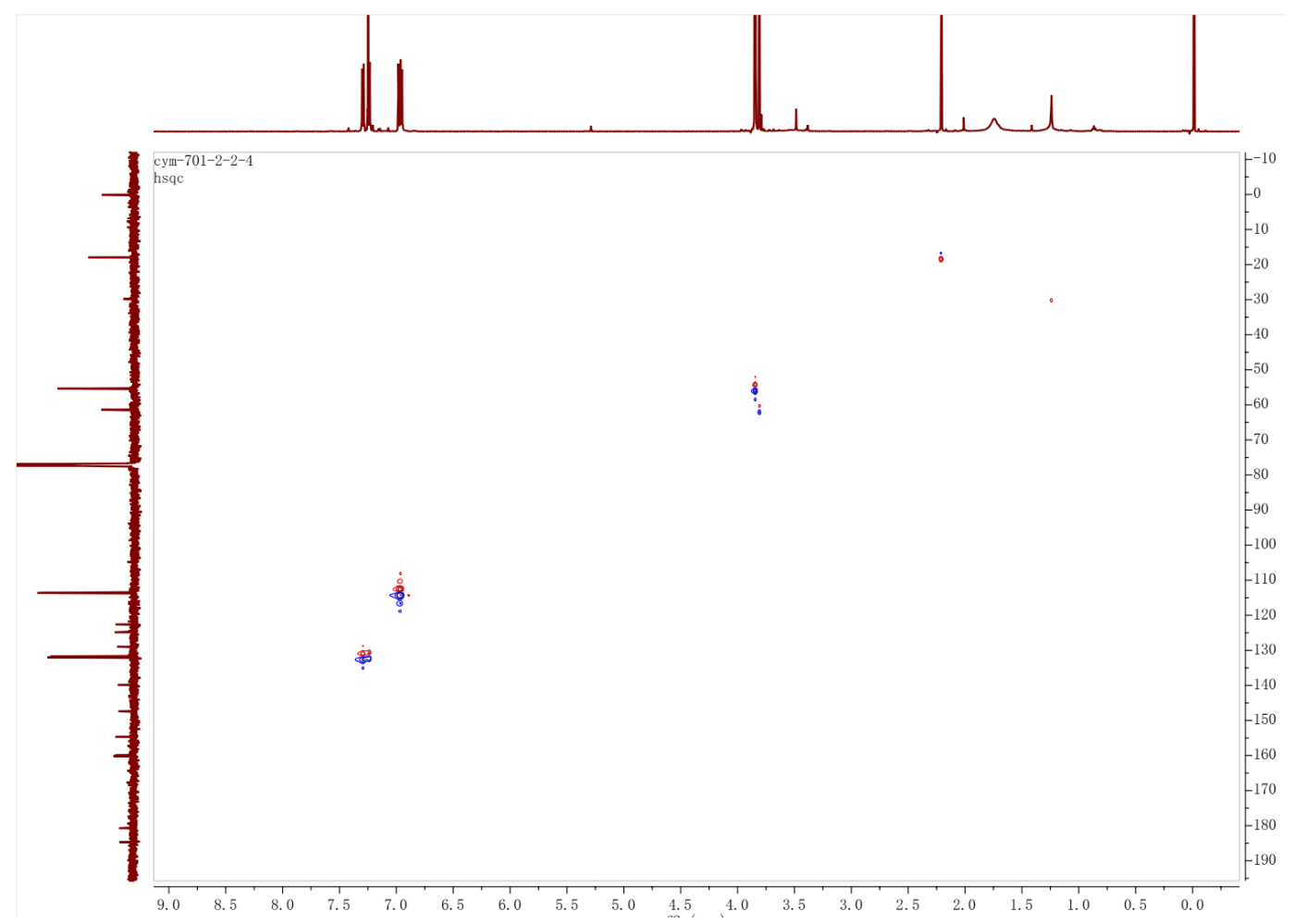


Figure S33. ${ }^{1} \mathrm{H}-{ }^{1} \mathrm{H}$ COSY spectrum of nocarterphenyl G (4).

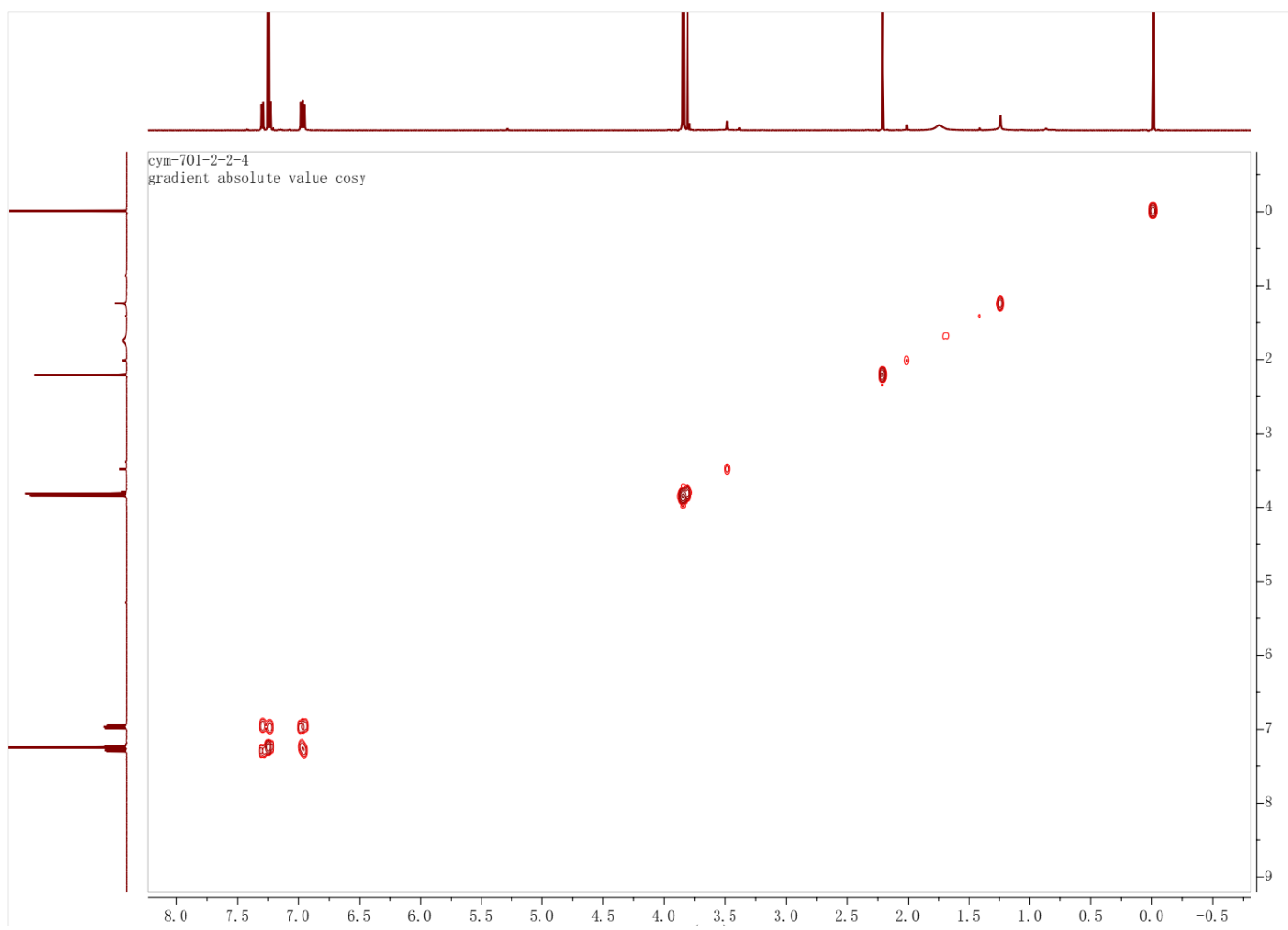

Figure S34. ${ }^{1} \mathrm{H}^{-13} \mathrm{C}$ HMBC spectrum of nocarterphenyl $\mathrm{G}(4)$.

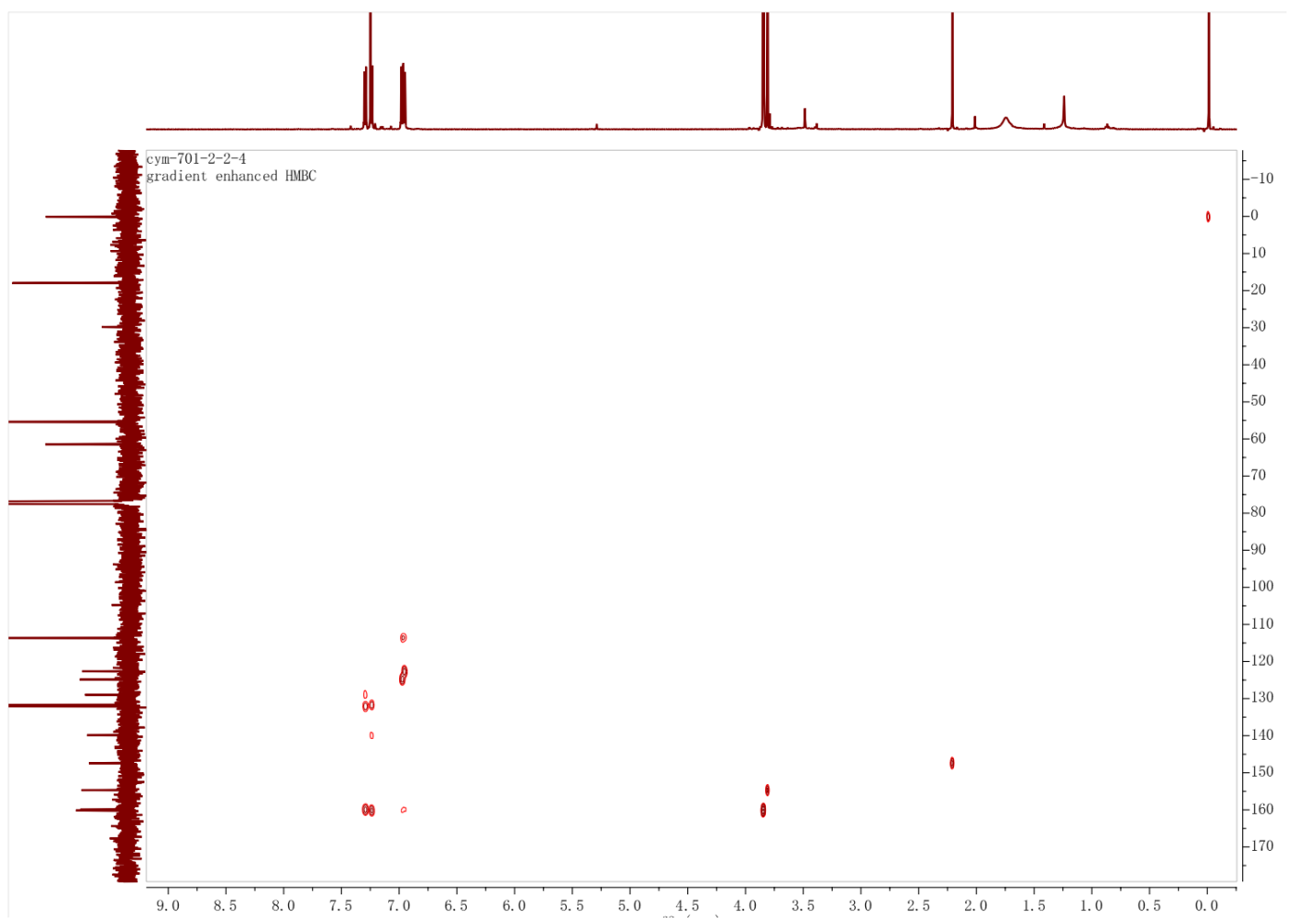

21 
Figure S35. HRESIMS spectrum of nocarterphenyl G (4).

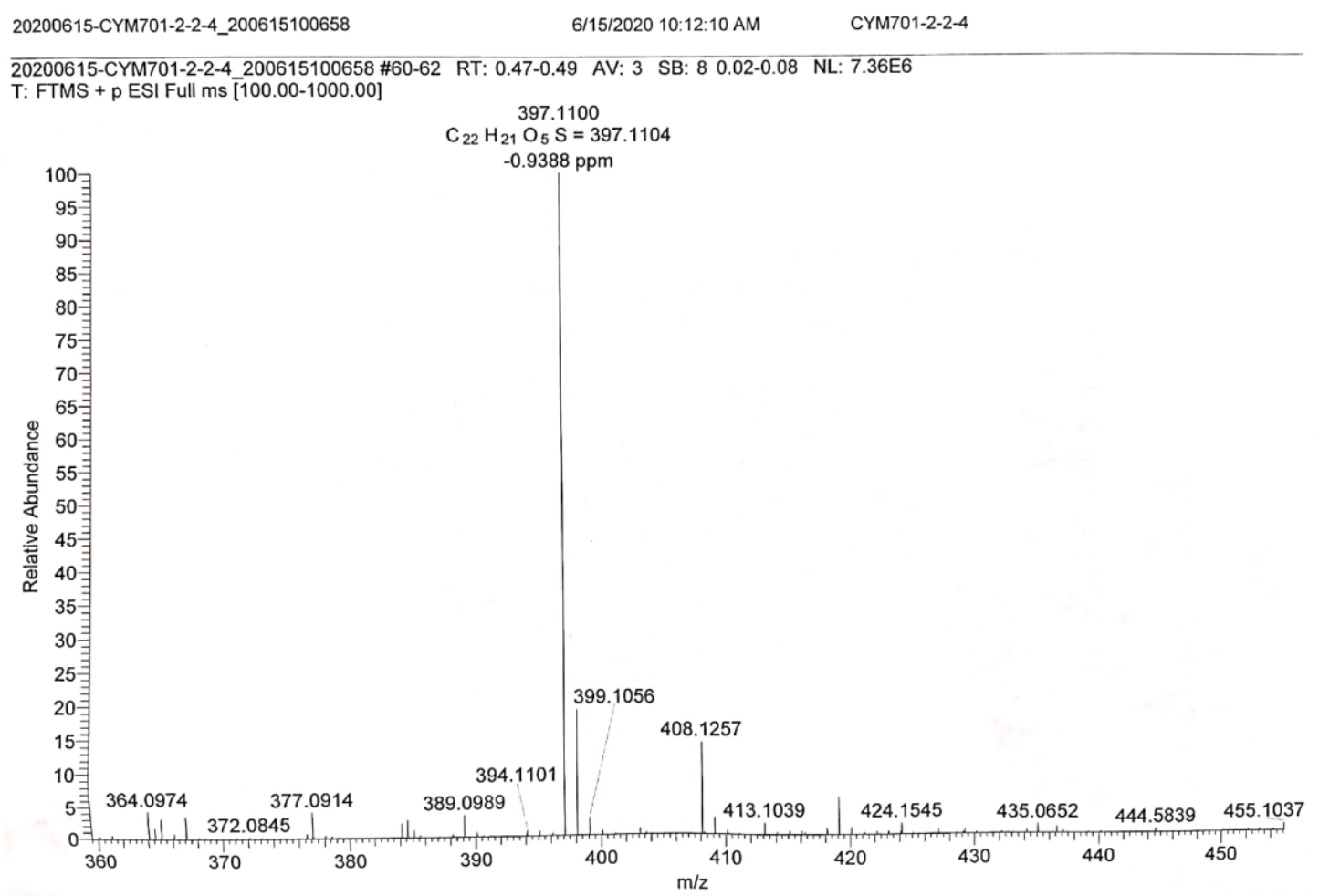

Figure S36. IR spectrum of nocarterphenyl G (4).

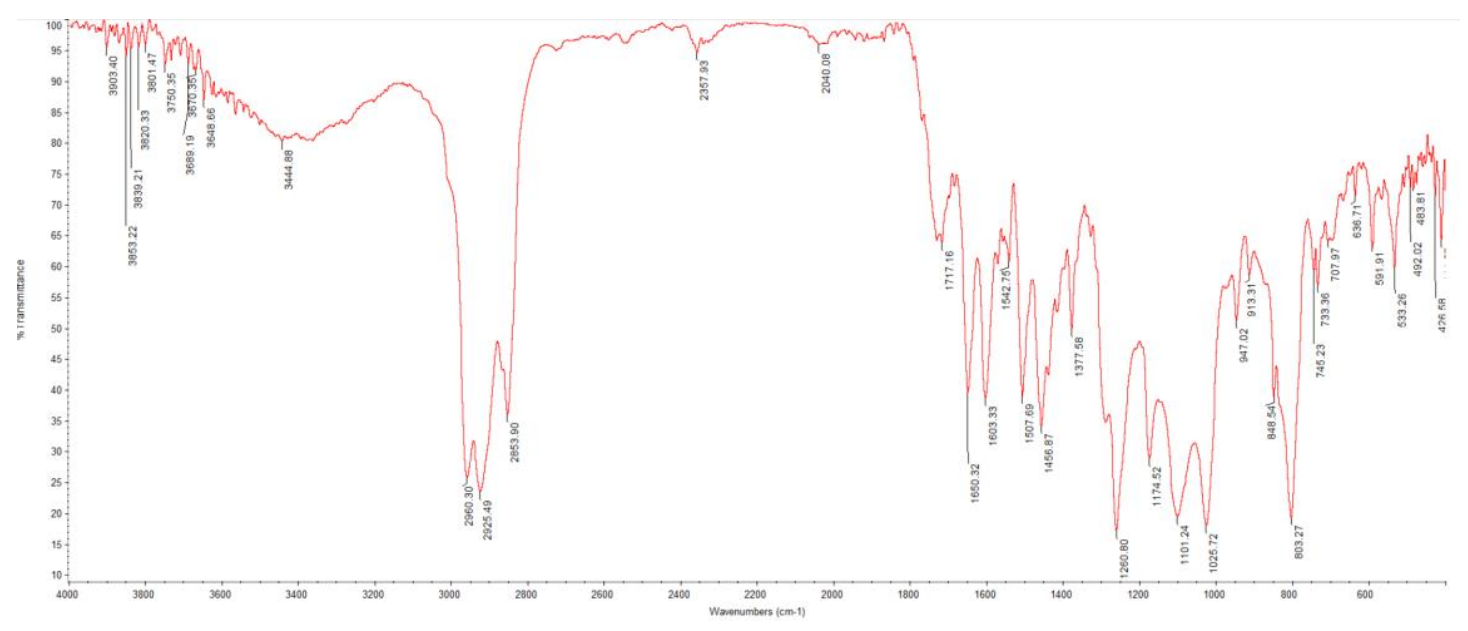


Figure S37. ${ }^{1} \mathrm{H}$ NMR (400 MHz, $\mathrm{CDCl}_{3}$ ) spectrum of nocarterphenyl H (5).

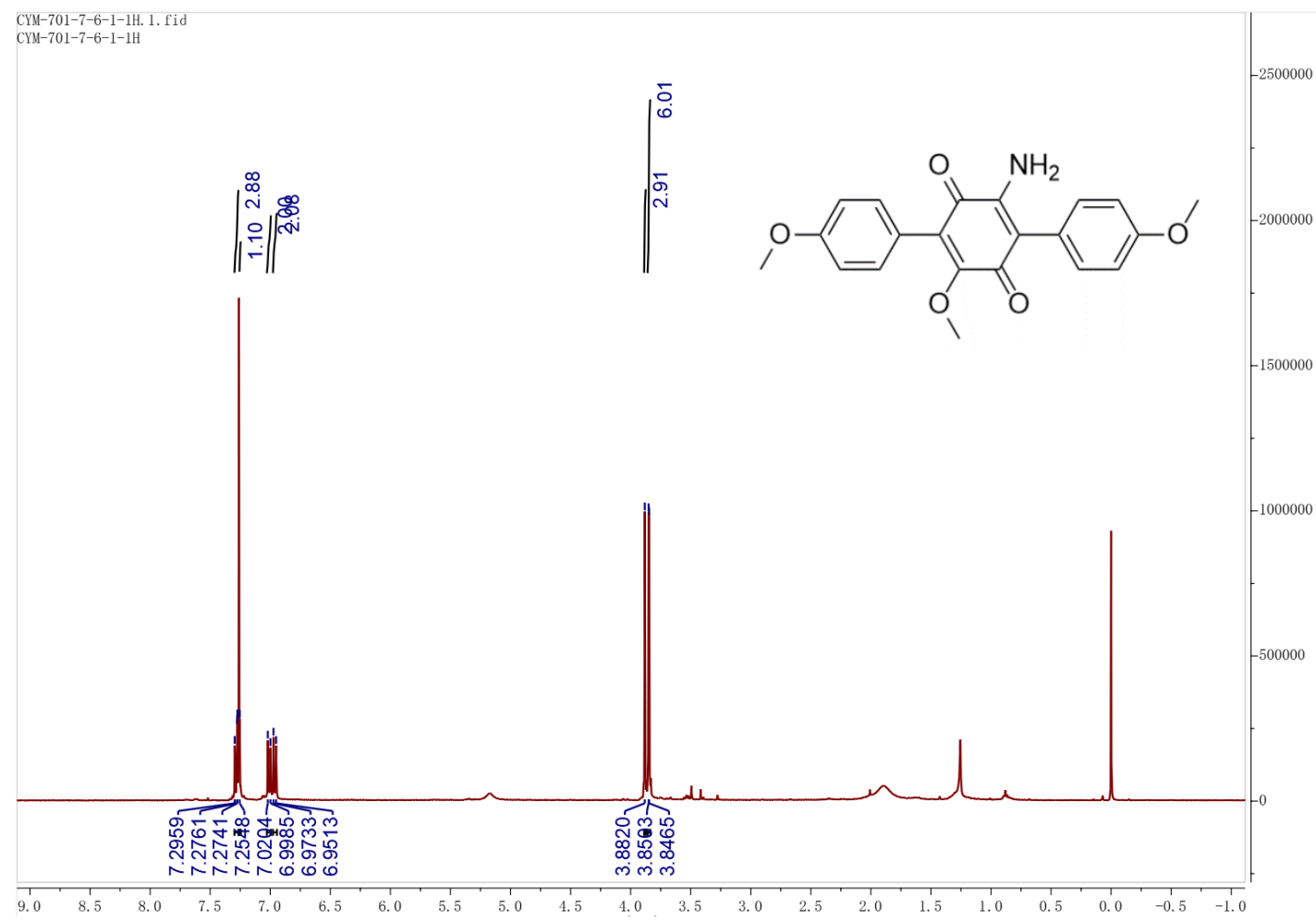

Figure S38. ${ }^{13} \mathrm{C}$ NMR $\left(125 \mathrm{MHz}, \mathrm{CDCl}_{3}\right)$ spectrum of nocarterphenyl $\mathrm{H}(\mathbf{5})$.

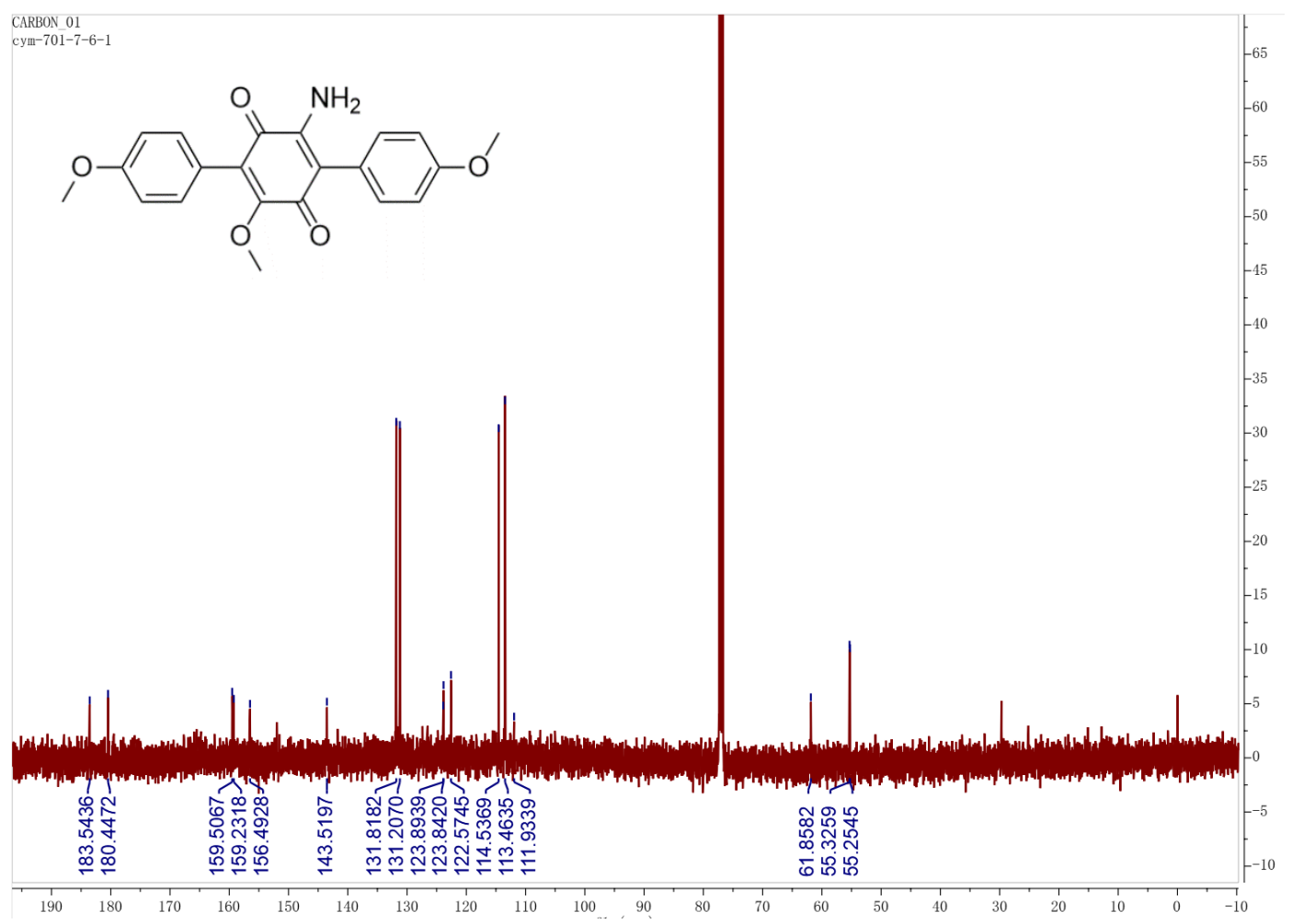


Figure S39. HSQC spectrum of nocarterphenyl H (5).

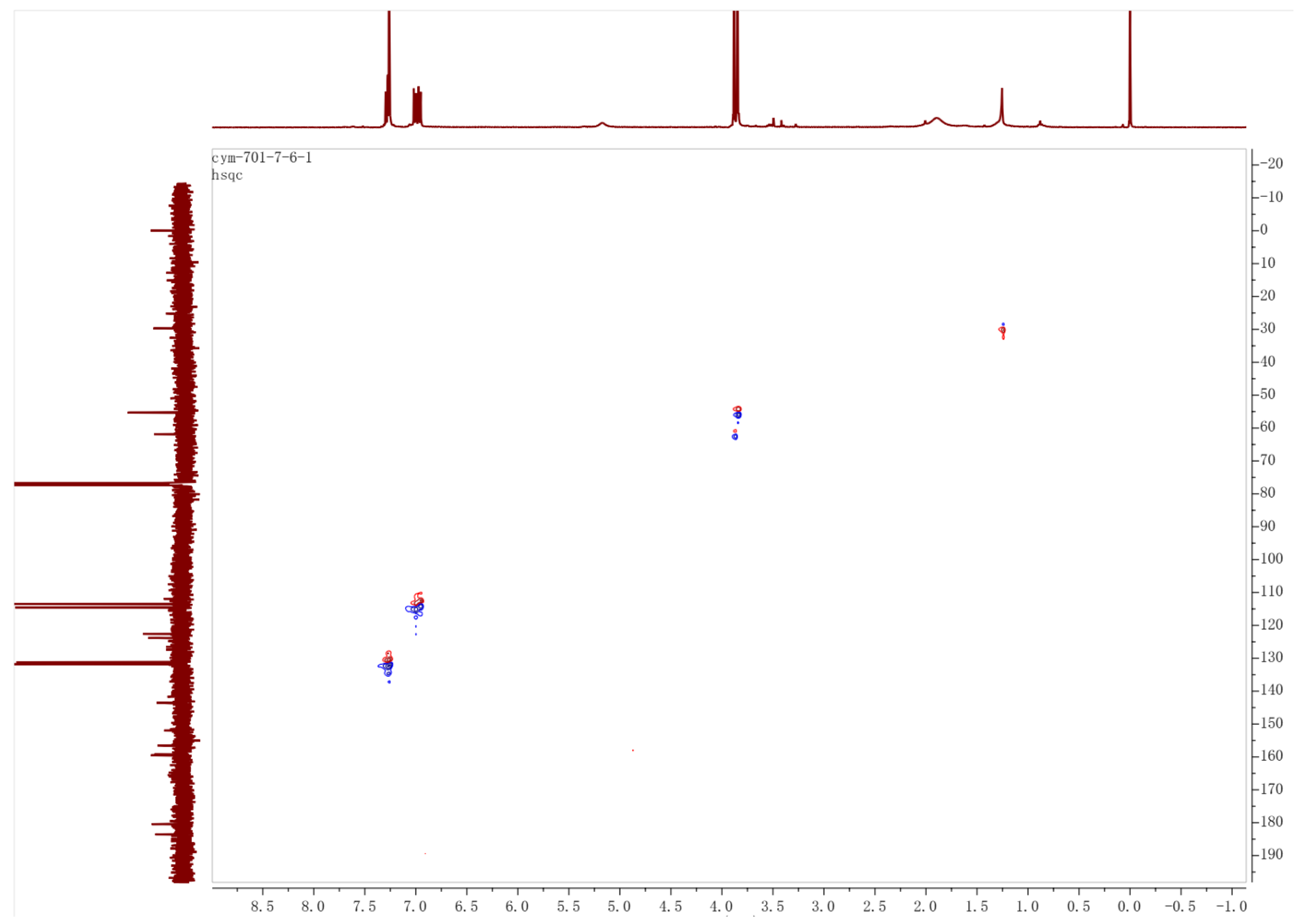

Figure S40. ${ }^{1} \mathrm{H}-{ }^{1} \mathrm{H}$ COSY spectrum of nocarterphenyl H (5).

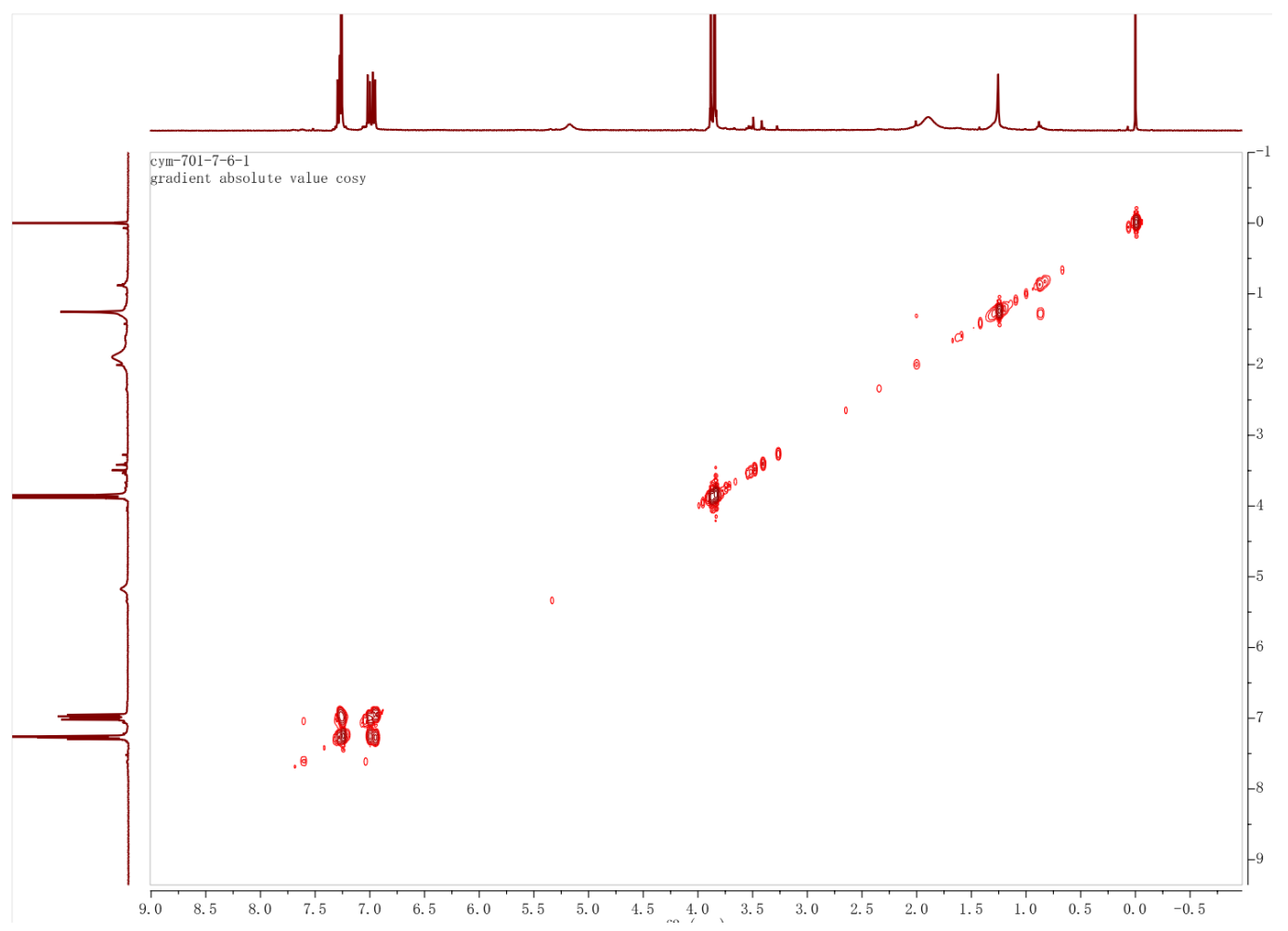

24 
Figure S41. ${ }^{1} \mathrm{H}-{ }^{13} \mathrm{C}$ HMBC spectrum of nocarterphenyl H (5).

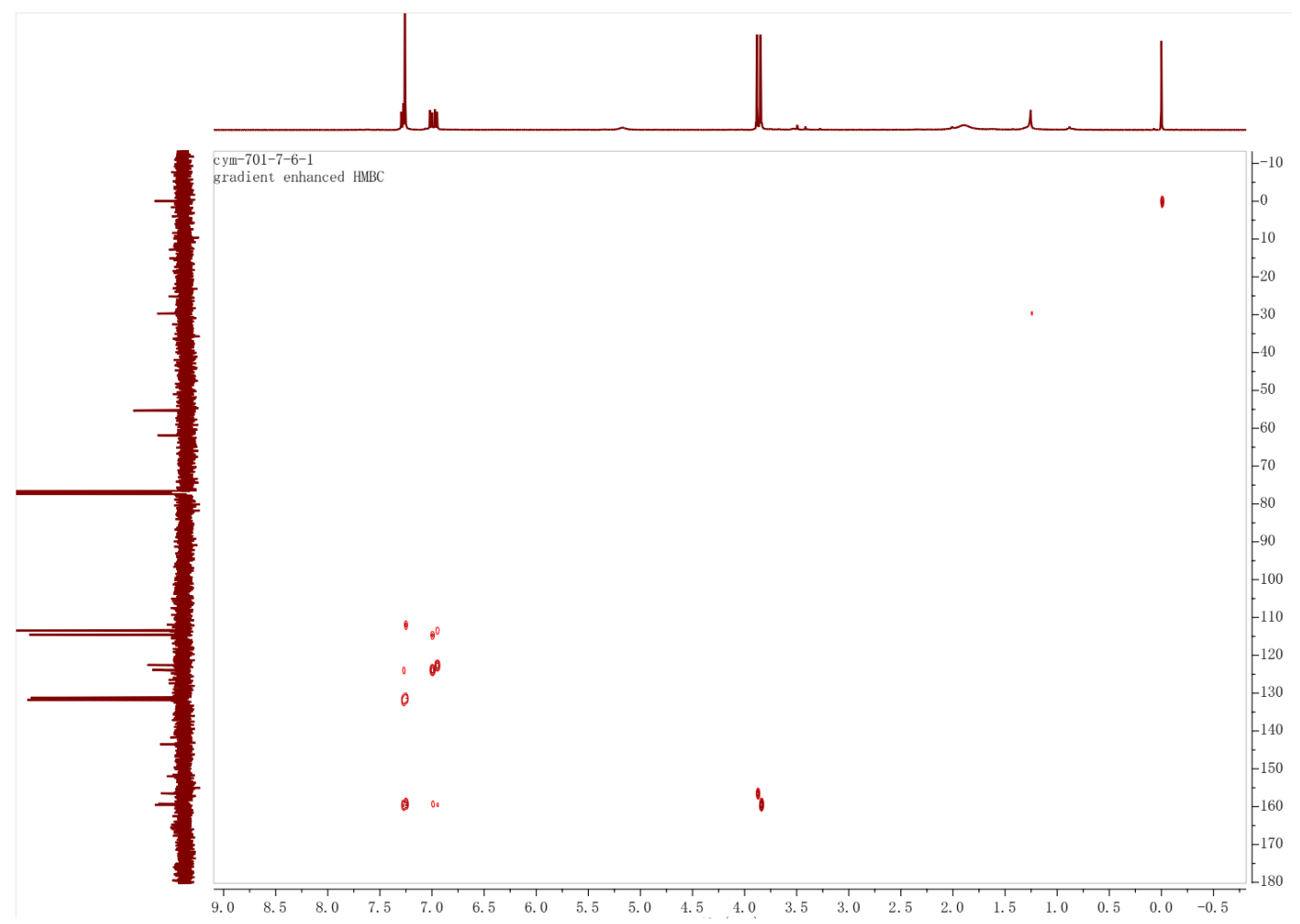

Figure S42. HRESIMS spectrum of nocarterphenyl H (5).

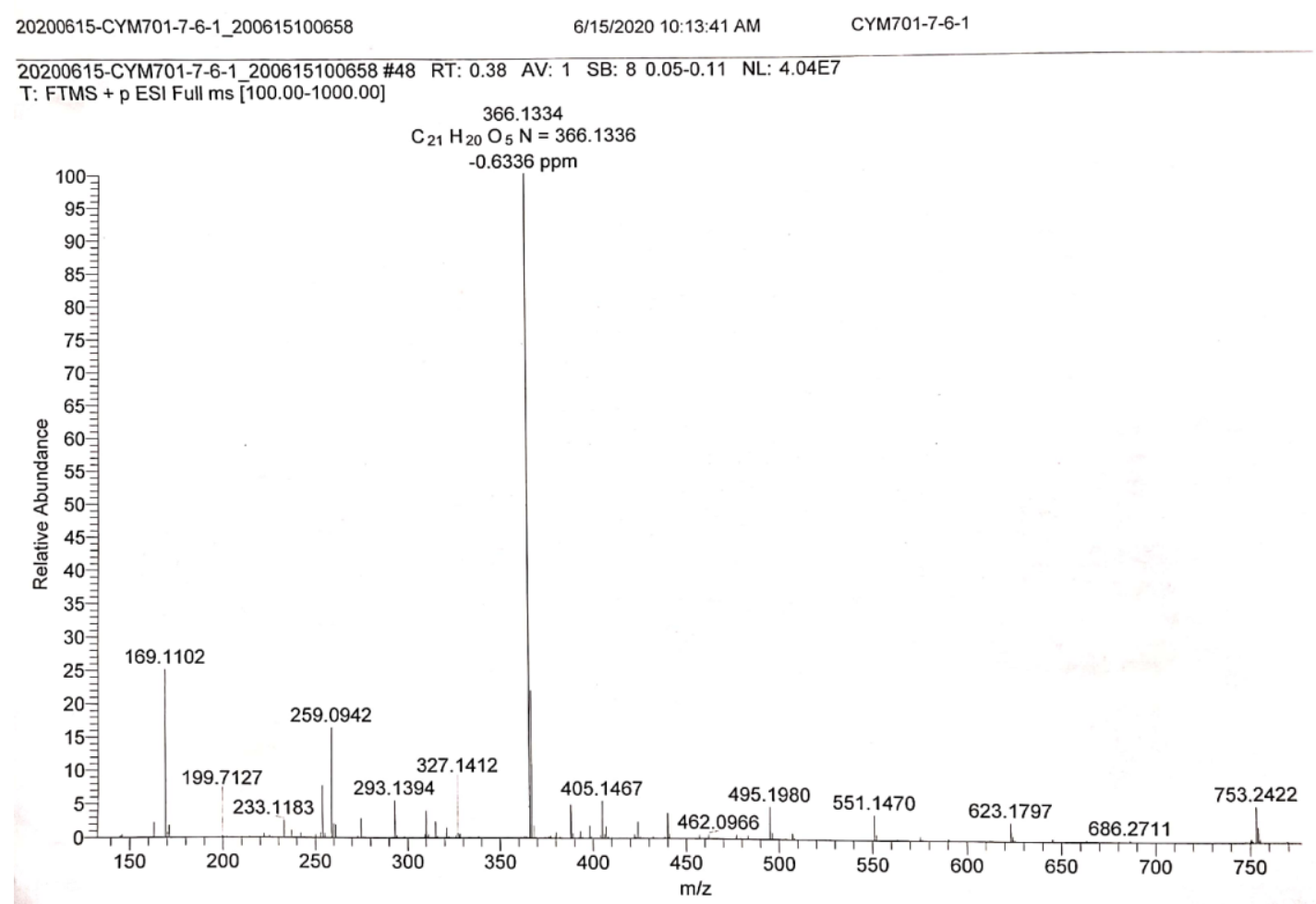


Figure S43. IR spectrum of nocarterphenyl H (5).

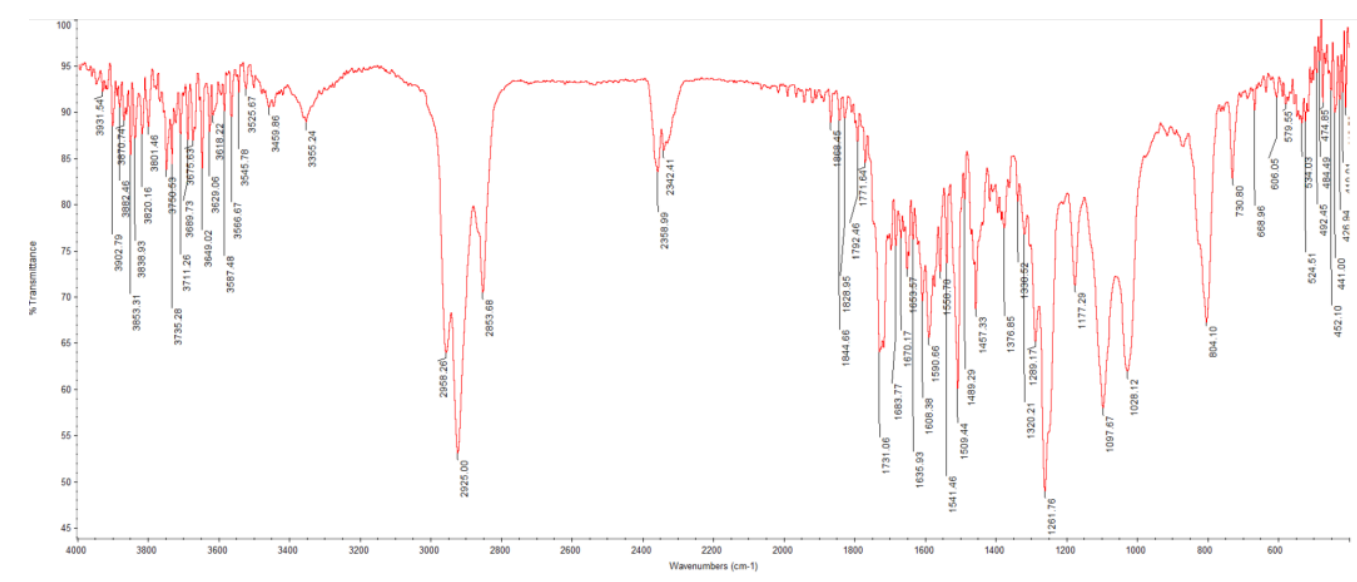

Figure S44. Key HMBC and COSY correlations of nocarterphenyl H (5).

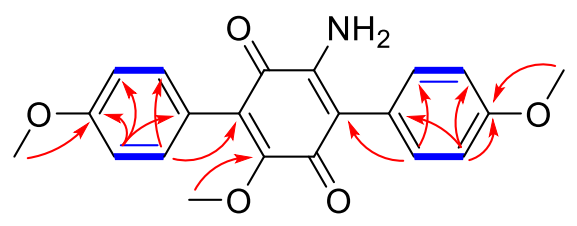

5

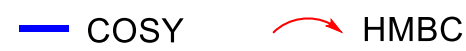

Figure S45. Plausible biogenetic pathway of nocarterphenyls D-H (1-5).

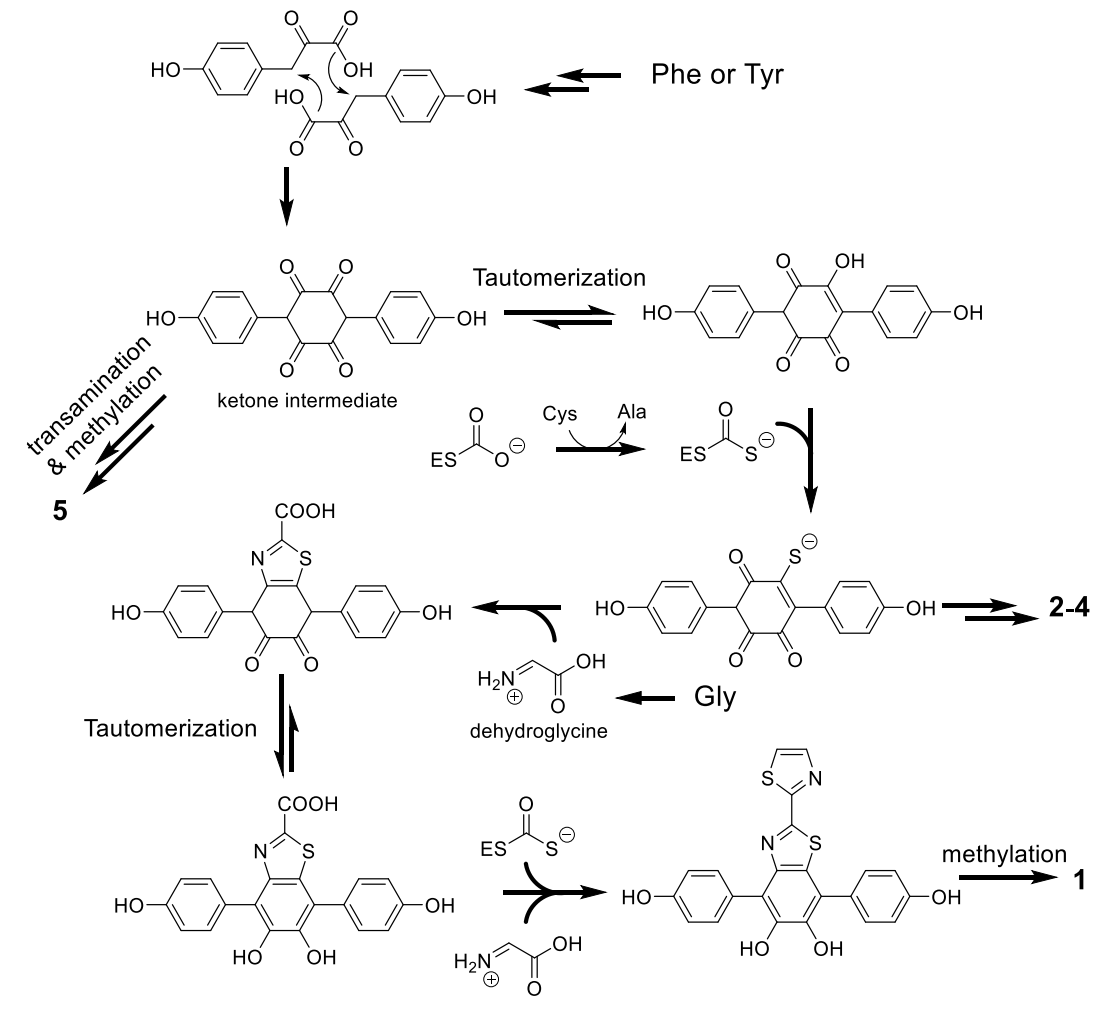


Table S1. ${ }^{13} \mathrm{C}$ NMR of 5-methoxy-4,7-bis(4-methoxyphenyl)benzo[ $\left.d\right]$ thiazol-6-ol and nocarterphenyl D (1) in $\mathrm{CDCl}_{3}$ ( $\delta$ in ppm)

\begin{tabular}{lll}
\hline & 5-methoxy-4,7-bis(4-methoxyphenyl)benzo[d]thiazol-6-ol & nocarterphenyl D (1) \\
\cline { 2 - 3 } No. & $\delta_{\mathrm{C}}$ & $\delta_{\mathrm{C}}$ \\
\hline 1 & $126.6, \mathrm{C}$ & $126.2, \mathrm{C}$ \\
$2 / 6$ & $131.6, \mathrm{CH}$ & $132.1, \mathrm{CH}$ \\
$3 / 5$ & $113.9, \mathrm{CH}$ & $113.5, \mathrm{CH}$ \\
4 & $159.4, \mathrm{C}$ & $159.3, \mathrm{C}$ \\
7 & $151.6, \mathrm{CH}$ & $158.2, \mathrm{C}$ \\
8 & & $162.3, \mathrm{C}$ \\
9 & & $143.9, \mathrm{CH}$ \\
10 & & $121.4, \mathrm{CH}$ \\
$1^{\prime}$ & $126.8, \mathrm{C}$ & $126.7, \mathrm{C}$ \\
$2^{\prime}$ & $144.6, \mathrm{C}$ & $144.8, \mathrm{C}$ \\
$3^{\prime}$ & $144.7, \mathrm{C}$ & $145.6, \mathrm{C}$ \\
$4^{\prime}$ & $119.0, \mathrm{C}$ & $119.0, \mathrm{C}$ \\
$5^{\prime}$ & $131.8, \mathrm{C}$ & $133.9, \mathrm{C}$ \\
$6^{\prime}$ & $145.4, \mathrm{C}$ & $145.3, \mathrm{C}$ \\
$1^{\prime \prime}$ & $128.7, \mathrm{C}$ & $128.3, \mathrm{C}$ \\
$2^{\prime \prime} / 6 "$ & $130.3, \mathrm{CH}$ & $130.3, \mathrm{CH}$ \\
$3^{\prime \prime} / 5 "$ & $114.2, \mathrm{CH}$ & $114.2, \mathrm{CH}$ \\
$4^{\prime \prime}$ & $159.4, \mathrm{C}$ & $159.4, \mathrm{C}$ \\
$4-\mathrm{OCH}_{3}$ & $55.3, \mathrm{CH}$ & $55.3, \mathrm{CH} 3$ \\
$2^{\prime}-\mathrm{OCH}_{3}$ & $61.1, \mathrm{CH}$ & $60.9, \mathrm{CH}$ \\
$4^{\prime}-\mathrm{OCH}_{3}$ & $55.3, \mathrm{CH} 3$ & $55.4, \mathrm{CH}$ \\
\hline & &
\end{tabular}

